\title{
REPRESENTATIONS OF REDUCTIVE GROUPS DISTINGUISHED BY SYMMETRIC SUBGROUPS
}

\author{
ITAY GLAZER
}

\begin{abstract}
Let $G$ be a complex connected reductive group, $G^{\theta}$ be its fixed point subgroup under a Galois involution $\theta$ and $H$ be an open subgroup of $G^{\theta}$. We show that any $H$-distinguished representation $\pi$ satisfies:

1) $\pi^{\theta} \simeq \tilde{\pi}$, where $\tilde{\pi}$ is the contragredient representation and $\pi^{\theta}$ is the twist of $\pi$ under $\theta$.

2) $\operatorname{dim}_{\mathbb{C}}\left(\pi^{*}\right)^{H} \leq|B \backslash G / H|$, where $B$ is a Borel subgroup of $G$.

By proving the first statement, we give a partial answer to a conjecture by Prasad and Lapid.
\end{abstract}

\section{Contents}

1. Introduction

1.1. Main results

1.2. Structure of the paper

2. Preliminaries

2.1. Basic definitions

2.2. Some tools from the theory of distributions

3. The complex version of the Langlands classification $\quad 8$

4. On the geometry of $B$-orbits on $G / H \quad 10$

5. Some reductions using tools from the theory of distributions 12

6. Proof of the main theorem- Theorem $1.3 \quad 14$

6.1. Calculation of $N_{B(n), n}^{Q^{\prime}} \quad 15$

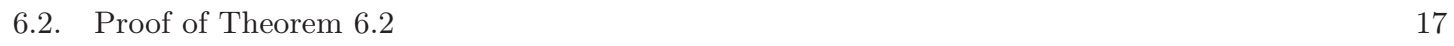

$\begin{array}{lll}\text { Appendix A. Langlands' classification } & 20\end{array}$

$\begin{array}{lll}\text { A.1. } & \text { Proof of Theorem } 3.1 & 21\end{array}$

A.2. Proof of Corollary 3.4 23

Appendix B. Some facts about the structure of real reductive groups 24

$\begin{array}{ll}\text { B.1. Parabolic subgroups } & 25\end{array}$

$\begin{array}{lll}\text { Appendix C. } & \text { Restriction of scalars and Galois involution } & 27\end{array}$

References 


\section{INTRODUCTION}

Let $\underline{G}$ be a connected reductive algebraic group defined over $\mathbb{R}, \underline{G}^{\theta}$ be its fixed point subgroup under some involution $\theta$ defined over $\mathbb{R}, G=\underline{G}(\mathbb{R})$ and $G^{\theta}=\underline{G}^{\theta}(\mathbb{R})$. We call $(G, H)$ a real symmetric pair if $H$ is an open subgroup of $G^{\theta}$. Denote by $\mathcal{S} \mathcal{A F}(G)$ the category of all finitely generated, smooth, admissible, moderate growth, Fréchet representations of $G$ with continuous linear $G$-maps as morphisms. Denote by $\mathcal{S} \mathcal{A F}_{\text {Irr }}(G)$ the set of irreducible objects in $\mathcal{S} \mathcal{A} \mathcal{F}(G)$. A fundamental task in the representation theory of the pair $(G, H)$ is to explore representations $\pi \in \mathcal{S} \mathcal{A} \mathcal{F}_{\operatorname{Irr}}(G)$ embedded in $C^{\infty}(G / H)$. By Frobenius reciprocity, the irreducible representations embedded in $C^{\infty}(G / H)=\operatorname{Ind}_{H}^{G}(\mathbb{C})$ are exactly the irreducible representations $\pi$ with a non trivial $H$-invariant continuous functional, i.e $\pi$ with $\left(\pi^{*}\right)^{H} \neq 0$. Such representations are called H-distinguished. Hence, when studying the symmetric pair $(G, H)$, we would like to answer the following questions:

(1) Which representations $\pi \in \mathcal{S A F}_{\text {Irr }}(G)$ are $H$-distinguished?

(2) What can we say about the multiplicity of such representation $\pi$, i.e, the number $\operatorname{dim}_{\mathbb{C}}\left(\pi^{*}\right)^{H}$ ?

The first step towards answering the first question is to find a necessary condition for a representation $\pi$ to be $H$-distinguished. The following theorem is a version of a classical theorem of Gelfand and Kazhdan (see [GK75]) and it suggests a certain candidate for such a condition.

Theorem. (Special case of Gelfand-Kazhdan criterion) Let $(G, H)$ be a real symmetric pair, where $H=\underline{G}^{\theta}(\mathbb{R})$, and let $\sigma$ be the anti-involution $\sigma(g)=\theta(g)^{-1}$. Assume that $\sigma(\xi)=\xi$ for all bi $H$ invariant Schwartz distributions $\xi$ on $G$. Then any $H$-distinguished representation $\pi \in \mathcal{S A F}_{\operatorname{Irr}}(G)$ satisfies $\widetilde{\pi} \simeq \pi^{\theta}$, where $\widetilde{\pi}$ denotes the contragredient representation and $\pi^{\theta}$ is the twist of $\pi$ by $\theta$.

We see that the symmetric pairs $(G, H)$ that satisfy the above G-K criterion have the property:

$$
\left(\pi^{*}\right)^{H} \neq 0 \Longrightarrow \widetilde{\pi} \simeq \pi^{\theta}
$$

for any $\pi \in \mathcal{S} \mathcal{A F}_{\text {Irr }}(G)$. As many symmetric pairs satisfy the above $\mathrm{G}-\mathrm{K}$ criterion, it is therefore natural to ask for a symmetric pair $(G, H)$ if the condition $\tilde{\pi} \simeq \pi^{\theta}$ is necessary and sufficient for $\pi$ to be $H$-distinguished. Using (e.g. [AG09, Theorem 8.2.1]), one can deduce that this condition is not sufficient. Indeed, any representation $\pi \in \mathcal{S A F}_{\operatorname{Irr}}\left(\mathrm{GL}_{2 \mathrm{n}}(\mathbb{C})\right)$ satisfies $\tilde{\pi} \simeq \pi^{\theta}$, where $\theta(g):=\Omega^{-1}\left(g^{t}\right)^{-1} \Omega, \Omega=\left(\begin{array}{cc}0 & I_{n} \\ -I_{n} & 0\end{array}\right)$. In particular, representations that are not $\operatorname{Sp}_{2 \mathrm{n}}(\mathbb{C})$-distinguished satisfy $\tilde{\pi} \simeq \pi^{\theta}$ (see [GSS15] for existence of such representations).

Although the condition $\tilde{\pi} \simeq \pi^{\theta}$ is not sufficient, it is conjectured that it is necessary when $G$ is a complex reductive group.

Conjecture 1.1. Let $(G, H)$ be a real symmetric pair, where $G$ is a complex connected reductive group, and let $\pi \in \mathcal{S A F}_{\operatorname{Irr}}(G)$ be $H$-distinguished. Then $\tilde{\pi} \simeq \pi^{\theta}$.

This conjecture can be generalized to the following conjecture by Lapid: 
Conjecture 1.2. (Lapid) Let $\underline{G}$ be a connected algebraic group defined over a local field $F, \theta$ : $\underline{G} \longrightarrow \underline{G}$ an involution defined over $F, \underline{H}=\underline{G}^{\theta}$ and write $G=\underline{G}(F), H=\underline{H}(F)$. Let $\pi$ be either in $\mathcal{S} \mathcal{A F}_{\operatorname{Irr}}(G)$ in the Archimedean case, or irreducible and smooth in the non-Archimedean case. Assume that $\pi$ is $H$-distinguished, then the L-packet of $\pi$ is invariant with respect to the functor $\pi \longmapsto \tilde{\pi} \circ \theta$.

These conjectures have been proven for the pairs $\left(\mathrm{GL}_{\mathrm{n}}(\mathbb{C}), \mathrm{U}(p, q)\right)$ and their non-Archimedean analogue in [ALOF12], and for the pair $\left(\mathrm{GL}_{\mathrm{n}}(\mathbb{C}), \mathrm{GL}_{\mathrm{n}}(\mathbb{R})\right)$ in $[\mathrm{Kem} 15]$.

In [Pra], Prasad formulates several conjectures about $\underline{G}(F)$-distinguished representations of $\underline{G}(E)$ in terms of the Langlands parameters of the representations of $\underline{G}(E)$, where $E / F$ is a quadratic extension of local fields. He introduces a character $\omega_{G}: \underline{G}(F) \longrightarrow \mathbb{Z} / 2 \mathbb{Z}$ (see[Pra, Section 8]) that appears in many questions about distinction. Hence, it is sometimes more interesting to consider representations of $\underline{G}(E)$ that are $\left(\omega_{G}, \underline{G}(F)\right)$-distinguished. Conjecture 3 of [Pra] is part of the motivation to consider Galois pairs $(\underline{G}(\mathbb{C}), \underline{G}(\mathbb{R}))$ and in particular to discuss representations $\pi \in \mathcal{S A F}_{\operatorname{Irr}}(\underline{G}(\mathbb{C}))$ that are $(\omega, \underline{G}(\mathbb{R}))$-distinguished for any character $\omega$ that is trivial on the connected component $\underline{G}(\mathbb{R})^{0}$ of $\underline{G}(\mathbb{R})$. In this work we present a partial answer to Conjecture 1.1 and the first part of Conjecture 3 in [Pra].

A vast study has been done regarding the multiplicity of $H$-distinguished representations, which is the second question we are dealing in this paper. In [Ban87] it has been shown that the multiplicity $\operatorname{dim}_{\mathbb{C}}\left(\pi^{*}\right)^{H}$ is finite for real symmetric spaces. In [KO13, KS16, AGM16], several bounds on the multiplicities have been established for real spherical pairs. In the non-Archimedean case, finiteness of $\operatorname{dim}_{\mathbb{C}}\left(\pi^{*}\right)^{H}$ has been shown for symmetric pairs in [Del10] and certain spherical pairs in [SV].

1.1. Main results. We call $(G, H)$ a Galois symmetric pair if $G$ is obtained by restriction of scalars of the complexification of $G^{\theta}$ (e.g $G^{\theta}$ is a real form of $G$ ), and $H$ is an open subgroup of $G^{\theta}$. In this paper we prove Conjecture 1.1 and present a bound on $\operatorname{dim}_{\mathbb{C}}\left(\pi^{*}\right)^{H}$, for the case of Galois pairs $(G, H)$. We also deduce the first part of Conjecture 3 of [Pra], namely that for any $\pi \in \mathcal{S} \mathcal{A F}_{\operatorname{Irr}}(\underline{G}(\mathbb{C}))$ that is $\left(\omega_{G}, \underline{G}(\mathbb{R})\right)$-distinguished it holds that $\tilde{\pi} \simeq \pi^{\theta}$, where $\tilde{\pi}$ is the contragredient representation, $\theta$ is the Galois involution of $\underline{G}(\mathbb{C})$ fixing $\underline{G}(\mathbb{R})$ and $\pi^{\theta}$ is the twist of $\pi$ by $\theta$.

Although we are mostly interested in Galois pairs, we work in a slightly more general setting, where $G$ is a complex reductive group and $\theta$ is any real involution; let $\underline{G}$ be a connected reductive algebraic group defined over $\mathbb{R}$ and $\underline{G}_{\mathbb{C} / \mathbb{R}}$ be the restriction of scalars of the complexification of $\underline{G}$ (see Appendix C for more details) such that $G=\underline{G}(\mathbb{C})=\underline{G}_{\mathbb{C} / \mathbb{R}}(\mathbb{R})$. Let $\theta$ be an $\mathbb{R}$-involution of $\underline{G}_{\mathbb{C} / \mathbb{R}}$ and write $G^{\theta}=\underline{G}_{\mathbb{C} / \mathbb{R}}^{\theta}(\mathbb{R})$.

Remark. A motivating example for the above construction is the pair $\left(\mathrm{GL}_{\mathrm{n}}(\mathbb{C}), \mathrm{U}(n)\right)$, as the involution $\theta(g)=\left(g^{*}\right)^{-1}$ is defined over $\mathbb{R}$ only after we apply restriction of scalars to $\mathrm{GL}_{\mathrm{n}}$. 
We fix a Borel subgroup $B$, a $\theta$-stable maximal torus $T$, and a $\theta$-stable maximal $\mathbb{R}$-split torus $A$ in $G$, such that $A \subseteq T \subseteq B$ (see Lemma 5.1). Let $N_{G}(T)$ be the normalizer of $T$ in $G$ and $\delta_{B}$ be the modular character of $B$. We further assume that $\theta$ is an involution that satisfies the following condition $(\star)$

$$
\forall n \in N_{G}(T) \text { such that } \theta(n)=n^{-1} \text { we have that } \delta_{B^{\theta_{n}}}=\left.\delta_{B}^{1 / 2}\right|_{B^{\theta_{n}}},
$$

where $\theta_{n}$ is the involution of $G$ defined by $\theta_{n}(g)=n \theta(g) n^{-1}$ and $B^{\theta_{n}}$ is the fixed points subgroup of $B$ under $\theta_{n}$. We prove the following theorem:

Theorem 1.3. Let $G$ be a complex reductive group, $\theta$ be any involution of $G$ that satisfies property $(\star), H$ be an open subgroup of $G^{\theta}$ and $\pi \in \mathcal{S A F}_{\text {Irr }}(G)$ be $H$-distinguished. Then:

a) $\tilde{\pi} \simeq \pi^{\theta}$.

b) $\operatorname{dim}_{\mathbb{C}}\left(\pi^{*}\right)^{H} \leq|B \backslash G / H|$.

c) In particular, a) and b) holds for Galois pairs.

Part c) follows from a) and b) using the fact that any Galois involution satisfies $(\star)$ (see Theorem C.1).

Replacing $\underline{G}(\mathbb{R})$ with $\operatorname{ker}\left(\omega_{G}\right)$, the following corollary now easily follows:

Corollary 1.4. (Conjecture 3 of $[\mathrm{Pra}]$ ) Let $\underline{G}$ be a connected reductive algebraic group defined over $\mathbb{R}$ and $\omega_{G}: \underline{G}(\mathbb{R}) \longrightarrow \mathbb{Z} / 2 \mathbb{Z}$ be the character defined in [Pra, Section 8]. Then for any $\pi \in \mathcal{S A F}_{\operatorname{Irr}}(\underline{G}(\mathbb{C}))$ that is $\left(\omega_{G}, \underline{G}(\mathbb{R})\right)$-distinguished we have $\widetilde{\pi} \simeq \pi^{\theta}$.

In particular, we see that the symmetry condition $\tilde{\pi} \simeq \pi^{\theta}$ is not sensitive to distinction up to characters with finite image, such as $\omega_{G}$, although the property of distinction of representations is itself sensitive to such characters (e.g, the Steinberg representation of $\mathrm{PGL}_{2}(\mathbb{C})$ is not distinguished but only $\omega_{G}$-distinguished).

1.2. Structure of the paper. We prove Theorem 1.3 in several steps. In the first step we translate the problem from the language of representations to the language of invariant distributions. We use the complex version of the Langlands' classification to present a given representation $\pi$ as the unique quotient of $\operatorname{Ind}_{B}^{G}(\chi)$, where $\chi$ is some dominant character (see Theorem 3.1), and to deduce the following:

Theorem. (Step 1-Corollary 3.7) Let $\pi \in \mathcal{S} \mathcal{A F}_{\operatorname{Irr}}(G)$. Then $\operatorname{dim}_{\mathbb{C}}\left(\pi^{*}\right)^{H} \leq \operatorname{dim}_{\mathbb{C}} \mathcal{S}^{*}(G / H)^{B, \chi \cdot \delta_{B}^{-1 / 2}}$, where $\chi$ is the Langlands parameter of $\pi$ (see Theorem 3.1), and $\mathcal{S}^{*}(X)$ denotes the space of Schwartz distributions on a manifold $X$ (see Section 2.2).

The next step is to use some geometric results about $B$-orbits on $G / H$ (see Section 4, [Sp85] and [HW93]), together with some tools from the theory of distributions (see Section 2.2), to reduce the problem to a question about a single $B$-orbit. More precisely, we prove the following theorem: 
Theorem. (Step 2- Theorem 5.2) If $\mathcal{S}^{*}(G / H)^{B, \chi \cdot \delta_{B}^{-1 / 2}} \neq 0$ then there exists $n \in N_{G}(T)$ such that $\theta(n)=n^{-1}$ and:

$$
\left.\operatorname{dim}_{\mathbb{C}}\left(\operatorname{Sym}\left(N_{B(n), n}^{Q^{\prime}}\right) \otimes_{\mathbb{R}} \mathbb{C}\right)^{T^{\theta_{n}}, \chi}\right)>0,
$$

where $Q^{\prime}=\left\{g \in G \mid \theta(g)=g^{-1}\right\}, B(n)$ is the $B$ orbit of $n \in Q^{\prime} \cap N_{G}(T)$, and $T^{\theta_{n}}$ is the fixed points subgroup of $T$ under $\theta_{n}$.

Finally, by direct calculations, we decompose $\left.\operatorname{Sym}\left(N_{B(n), n}^{Q^{\prime}}\right) \otimes_{R} \mathbb{C}\right)^{T^{\theta_{n}}}, \chi$ into eigenspaces of $T^{\theta_{n}}$ that correspond to some negative weights and use the fact that $\chi$ is dominant to deduce the following theorem, which implies Theorem 1.3 a) and b).

Theorem. (Step 3- Theorem 6.2) If there exists $n \in N_{G}(T)$ such that $\theta(n)=n^{-1}$ and:

$$
\left.\operatorname{dim}_{\mathbb{C}}\left(\operatorname{Sym}\left(N_{B(n), n}^{Q^{\prime}}\right) \otimes_{\mathbb{R}} \mathbb{C}\right)^{T^{\theta_{n}}, \chi}\right)>0,
$$

then $\left.\operatorname{dim}_{\mathbb{C}}\left(\operatorname{Sym}\left(N_{B(n), n}^{Q^{\prime}}\right) \otimes_{R} \mathbb{C}\right)^{T_{n}, \chi}\right)=1$ and $\tilde{\pi} \simeq \theta \circ \pi$.

In Section 2 we present some basic definitions and tools from the theory of distributions. In Section 3 we introduce the complex version of Langlands' classification and some corollaries, including the proof of the first step (Corollary 3.7). In Section 4 we present several results from [Sp85] and [HW93] on $B$-orbits on $G / H$. In Section 5 we make several reductions using the tools in Section 2.2 and prove Theorem 5.2- the second step. In Section 6 we prove the third step and finish the proof of Theorem 1.3.

Acknowledgement. I would like to thank my advisors Avraham Aizenbud and Dmitry Gourevitch for presenting me with this problem, teaching and helping me in this work. I hold many thanks to Dipendra Prasad for introducing me with his work and for a very fruitful discussion and enlightening remarks. I also thank Erez Lapid, Yotam Hendel, Shachar Carmeli and Arie Levit for helpful discussions. I was partially supported by ISF grant 687/13, ISF grant 756/12, ERC StG 637912 and by Minerva foundation grant.

\section{PRELiminaries}

2.1. Basic definitions. A reductive algebraic group is an algebraic group $\underline{G}$ over an algebraically closed field such that the unipotent radical of $\underline{G}$ is trivial. A real reductive group $G$ is the real points $G=\underline{G}(\mathbb{R})$ of an algebraic group $\underline{G}$ defined over $\mathbb{R}$, where $\underline{G}(\mathbb{C})$ is reductive. The group $\underline{G}(\mathbb{R})$ inherits the natural topology from $\mathbb{R}^{N}$ and this gives $\underline{G}(\mathbb{R})$ a structure of a Lie group.

Let $G=\underline{G}(\mathbb{R})$ be a real reductive group and $H$ a closed subgroup. A pair $(G, H)$ is called a real symmetric pair if there exists an involution $\theta$ of $\underline{G}$ such that $H$ is an open subgroup of $\underline{G}^{\theta}(\mathbb{R})$ of $\theta$-invariant elements.

A Fréchet representation $(\pi, V)$ of $G$ over $\mathbb{C}$ is a morphism of groups $\pi: G \rightarrow A u t_{\mathbb{C}}(V)$, such that $V$ is a Fréchet $\mathbb{C}$-vector space, and that $G \times V \rightarrow V$ is continuous. A representation $(\pi, V)$ of 
$G$ over $\mathbb{C}$ is called irreducible if $V \neq 0$ and if $V$ has no proper, closed $G$-invariant subspace. A map $f: G \rightarrow V$ is called smooth if it has continuous partial derivatives of all orders, where the derivatives and their continuity are with respect to the Lie structure of $G$. A vector $v \in V$ is called smooth if its orbit map $c_{v}: G \rightarrow V$ defined by $c_{v}(g)=\pi(g) v$ is a smooth map.

A representation $(\pi, V)$ of $G$ over $\mathbb{C}$ is called:

- Smooth, if every $v \in V$ is smooth.

- Admissible, if for every maximal compact subgroup $K$ and every irreducible representation $\rho$ of $K, \rho$ has finite multiplicity in $\left.\pi\right|_{K}$.

If $(\pi, V)$ is a smooth representation, the dual representation $\left(\pi^{*}, V^{*}\right)$ is defined by $V^{*}:=\operatorname{Hom}_{\mathbb{C}}(V, \mathbb{C})$ with the usual left $G$-action $g(f)(v)=f\left(g^{-1} v\right)$. It may not be smooth, and also $V^{*}$ is not necessarily a Fréchet space. Thus, we define $V^{* \infty}:=C_{c}^{\infty}(G)\left(V^{*}\right)$ where $C_{c}^{\infty}(G)$ acts on $V^{*}$ by $f(\varphi)(v)=\int_{G} f(g) \cdot\left\langle\pi^{*}(g)(\varphi), v\right\rangle d g$, where $f \in C_{c}^{\infty}(G)$ and $\varphi \in V^{*}$. This space is called the Garding space and it is a Fréchet space consisting of smooth vectors, so if we denote $\tilde{V}:=V^{* \infty}$, this defines a smooth representation $(\tilde{\pi}, \tilde{V})$ called the contragredient representation. A smooth representation $(\pi, V)$ of $G$ such that $\left(\pi^{*}\right)^{H} \neq 0$ for a subgroup $H$, is called $H$-distinguished.

Assume that $G$ is real reductive group and fix a maximal compact $K$. Fix a faithful algebraic representation $\rho: G \hookrightarrow \mathrm{GL}_{n}(\mathbb{R})$ and define $\|g\|:=\operatorname{Tr}\left(\rho(g) \cdot \rho(g)^{t}\right)+\operatorname{Tr}\left(\rho\left(g^{-1}\right), \rho\left(g^{-1}\right)^{t}\right)$. Let $(\pi, V)$ be a smooth Fréchet representation and denote by $\left\{P_{i}\right\}_{i \in I}$ the continuous semi-norms that define the Fréchet structure of $V$. We say that $(\pi, V)$ is of moderate growth if for any semi-norm $P_{i}$ there exists $P_{j}$ and $k(i) \in \mathbb{N}_{>0}$ such that:

$$
P_{i}(\pi(g) v) \leq\|g\|^{k(i)} \cdot P_{j}(v)
$$

for all $g \in G$ and $v \in V$ (see [BK14, 2.3] for more details). A vector $v \in(\pi, V)$ is called a $K$-finite vector if $\pi(K) v$ lies in a finite dimensional subspace of $V$. The space of $K$-finite vectors of $V$ is denoted $V_{K} .(\pi, V)$ is called finitely generated if $V_{K}$ is a finitely generated $\mathcal{U}(\mathfrak{g})$-module, where $\mathfrak{g}$ is the Lie algebra of $G$ and $\mathcal{U}(\mathfrak{g})$ is its universal enveloping algebra.

Putting everything together, we denote by $\mathcal{S} \mathcal{A F}(G)$ the category of all finitely generated, smooth, admissible, moderate growth, Fréchet representations of $G$ with continuous linear $G$-maps as morphisms.

2.2. Some tools from the theory of distributions. We will work with the notation and tools presented in [ALOF12, Appendix B]. For the convenience of the reader, we present notation and reformulations of some of the theorems that appear in [ALOF12, Appendix B], in a version better suited for this work. The results in this section holds for Nash manifolds, i.e smooth, semi-algebraic varieties. As any smooth algebraic variety can be endowed with a natural structure of a Nash manifold, we can apply the results presented in this section for smooth algebraic varieties . Let $G$ be an arbitrary group. 
- For any $G$-set $X$ and a point $x \in X$, we denote by $G(x)$ the $G$-orbit of $x$ and by $G_{x}$ the stabilizer of $x$.

- For any representation of $G$ on a vector space $V$ and a character $\chi$ of $G$, we denote by $V^{G, \chi}$ the subspace of $(G, \chi)$-equivariant vectors in $V$.

- Given manifolds $L \subseteq M$, we denote by $N_{L}^{M}:=\left(\left.T_{M}\right|_{L}\right) / T_{L}$ the normal bundle to $L$ in $M$ and by $C N_{L}^{M}:=\left(N_{L}^{M}\right)^{*}$ the conormal bundle. For any point $y \in L$, we denote by $N_{L, y}^{M}$ the normal space to $L$ in $M$ at the point $y$ and by $C N_{L, y}^{M}$ the conormal space to $L$ in $M$ at the point $y$.

- The symmetric algebra of a vector space $V$ will be denoted by $\operatorname{Sym}(V)=\oplus_{k \geq 0} \operatorname{Sym}^{\mathrm{k}}(V)$.

- The Fréchet space of Schwartz functions on a Nash manifold $X$ will be denoted by $\mathcal{S}(X)$ and the dual space of Schwartz distributions will be denoted by $\mathcal{S}^{*}(X)$.

- For any Nash vector bundle $E$ over $X$ we denote by $\mathcal{S}(X, E)$ the space of Schwartz sections of $E$ and by $\mathcal{S}^{*}(X, E)$ its dual space.

- For a closed subset $Z$ of a smooth manifold $X$ we set $\mathcal{S}_{Z}^{*}(X):=\left\{\xi \in \mathcal{S}^{*}(X): \operatorname{Supp}(\xi) \subseteq\right.$ $Z\}$.

Proposition 2.1. If $U$ is an open submanifold of $X$ then we have the following exact sequence:

$$
0 \longrightarrow \mathcal{S}_{X \backslash U}^{*}(X) \stackrel{i}{\longrightarrow} \mathcal{S}^{*}(X) \stackrel{e x t^{*}}{\longrightarrow} \mathcal{S}^{*}(U) \longrightarrow 0
$$

where $i: \mathcal{S}_{X \backslash U}^{*}(X) \hookrightarrow \mathcal{S}^{*}(X)$ is the natural inclusion and ext $*^{*} \mathcal{S}^{*}(X) \longrightarrow \mathcal{S}^{*}(U)$ is the dual map to extension by zero.

Definition. If $X$ is a smooth manifold and $G$ acts on $X$, then $X=\bigcup_{i=1}^{l} X_{i}$ is called a $G$ invariant stratification if all sets $X_{i}$ are $G$-invariant and $\cup_{i=1}^{j} X_{i}$ is open in $X$ for any $1 \leq j \leq l$.

Proposition 2.2. Let $G$ be a Nash group that acts on a Nash manifold $X$. Let $X=\cup_{i=1}^{l} X_{i}$ be a G-invariant stratification. Let $\chi$ be a character of $G$. Then:

$$
\operatorname{dim}_{\mathbb{C}}\left(\mathcal{S}^{*}(X)^{G, \chi}\right) \leq \sum_{i=1}^{l} \sum_{k=0}^{\infty} \operatorname{dim}_{\mathbb{C}}\left(\mathcal{S}^{*}\left(X_{i}, \operatorname{Sym}^{\mathrm{k}}\left(C N_{X_{i}}^{X}\right)\right)^{G, \chi}\right) .
$$

Proposition 2.3. Let $\underline{G}$ be an algebraic groups and $\underline{H}$ a closed subgroup. Set $G=\underline{G}(\mathbb{R}), H=$ $\underline{H}(\mathbb{R})$. Then $\mathcal{S}^{*}(G)^{H} \simeq \mathcal{S}^{*}(G / H)$.

Proposition 2.2 gives us a way to reduce from asking if a manifold $X$ has a non-trivial $(G, \chi)$ equivariant distribution to asking a similar question on a $G$-orbit. The next tool can help us to further reduce the problem:

Theorem 2.4. (Frobenius descent). Let a Nash group $G$ act transitively on a Nash manifold $Z$. Let $\varphi: X \rightarrow Z$ be a G-equivariant Nash map. Let $z \in Z$ and let $X_{z}$ be the fiber of $z$. Let $\chi$ be a tempered character of $G$ (see [AG08, Definition 5.1.1]). Then $\mathcal{S}^{*}(X)^{G, \chi}$ is canonically isomorphic 
to $\mathcal{S}^{*}\left(X_{z}\right)^{G_{z},\left.\chi \delta_{G}^{-1} \delta_{G}\right|_{G z}}$, where $G_{z}$ is the stabilizer of $z$ in $G$. Moreover, for any G-equivariant bundle $E$ on $X$, the space $\mathcal{S}^{*}(X, E)^{G, \chi}$ is canonically isomorphic to $\mathcal{S}^{*}\left(X_{z},\left.E\right|_{X_{z}}\right)^{G_{z},\left.\chi \delta_{G z}^{-1} \delta_{G}\right|_{G z}}$.

Corollary 2.5. Let a Nash group $G$ acts on a Nash manifold $X$. Let $X=\cup_{i=1}^{l} X_{i}$ be a G-invariant stratification. For each $i$, We have a canonical isomorphism:

$$
\left.\mathcal{S}^{*}\left(X_{i}, \operatorname{Sym}^{\mathrm{k}}\left(C N_{X_{i}}^{X}\right)\right)^{G, \chi} \simeq \mathcal{S}^{*}\left(\{x\}, \operatorname{Sym}^{\mathrm{k}}\left(C N_{X_{i}, x}^{X}\right)\right)^{G_{x}, \chi \delta_{G_{x}}^{-1} \delta_{G}}\right|_{G_{x}} .
$$

Proof. Choose $Z=X_{i}, I d: X_{i} \longrightarrow X_{i}$ and $z=x$ and $X_{z}=\{x\}$ and apply Theorem 2.4.

Corollary 2.6. Let a Nash group $G$ acts on a Nash manifold $X$. Let $X=\cup_{i=1}^{l} X_{i}$ be a G-invariant stratification. Then:

$$
\operatorname{dim}_{\mathbb{C}}\left(\mathcal{S}^{*}(X)^{G, \chi}\right) \leq \sum_{i=1}^{l} \sum_{k=0}^{\infty} \operatorname{dim}_{\mathbb{C}}\left(\left(\operatorname{Sym}^{\mathrm{k}}\left(N_{X_{i}, x}^{X}\right) \otimes_{\mathbb{R}} \mathbb{C}\right)^{G_{x},\left.\chi \delta_{G_{x}}^{-1} \delta_{G}\right|_{G_{x}}}\right) .
$$

Proof. Combine Proposition 2.2 with Corollary 2.5 and use the isomorphism

$$
\operatorname{Sym}^{\mathrm{k}}\left(N_{X_{i}, x}^{X}\right) \otimes_{\mathbb{R}} \mathbb{C} \simeq \mathcal{S}^{*}\left(\{x\}, \operatorname{Sym}^{\mathrm{k}}\left(C N_{X_{i}, x}^{X}\right)\right)
$$

\section{The COMPLEX VERSION OF THE LANGLANDS CLASSIFICATION}

The main goal of this section is to translate the property of being $H$-distinguished to the language of invariant distributions using the Langlands classification for complex reductive groups.

We assume that $G$ is a complex connected reductive group. Let $\mathfrak{g}$ be its Lie algebra, $K$ be a maximal compact subgroup of $G$ with Lie algebra $\mathfrak{k}, \tau$ the Cartan involution fixing $K$, and $\mathfrak{p}$ the -1 eigenspace of $\tau$. Let $\mathfrak{a}_{\mathfrak{p}}$ be a maximal abelian subspace of $\mathfrak{p}$, with the corresponding analytic subgroup $A_{\mathfrak{p}}$. Fix $A_{\mathfrak{p}} \subseteq A \subseteq T \subseteq B$, where $T$ is a maximal torus, $A$ is a maximal $\mathbb{R}$-split torus, and $B$ is a Borel subgroup that contains $T$.

The work of Zhelobenko and Naimark [ZN66] and Langlands [Lan73] provides the following known classification of representations $\pi \in \mathcal{S A F}_{\text {Irr }}(G)$ for a complex reductive group $G$ :

Theorem 3.1. Let $G$ be a connected complex reductive group and let $\pi \in \mathcal{S A F}_{\text {Irr }}(G)$. Then $\pi$ is the unique irreducible quotient of $\operatorname{Ind}_{B}^{G}(\chi)$, where

$$
\operatorname{Ind}_{B}^{G}(\chi)=\left\{f \in C^{\infty}(G) \mid \forall b \in B, f(b g)=\chi(b) \cdot \delta_{B}^{1 / 2}(b) \cdot f(g)\right\}=C^{\infty}(G)^{B, \chi^{-1} \delta_{B}^{-1 / 2}},
$$

and $\chi$ is a dominant character of $T$.

In Appendix A.1 we deduce Theorem 3.1 from the Langlands classification (see Theorem A.1), although historically, the Langlands classification appeared after the work of Zhelobenko and Naimark, who dealt with the case of a complex group. 
Set $W\left(G, A_{\mathfrak{p}}\right)$ and $W(G, T)$ to be the Weyl groups corresponding to the root systems $\Sigma\left(G, A_{\mathfrak{p}}\right)$ and $\Sigma(G, T)$. Note that the Weyl group $W(G, T) \simeq N_{G}(T) / T$ acts on $T$ by $w . t:=n t n^{-1}$, where $n \in N_{G}(T)$ and $w=n T$. Similarly $w \cdot \chi(t):=\chi\left(n^{-1} t n\right)$.

Proposition 3.2. Let $\pi_{1}=\operatorname{Ind}_{B}^{G}(\chi)$ and $\pi_{2}=\operatorname{Ind}_{B}^{G}\left(\chi^{\prime}\right)$ in $\mathcal{S} \mathcal{A F}(G)$, be unitary principal series representations of $G$. Then there exists an intertwining operator $L: \pi_{1} \longrightarrow \pi_{2}$ if and only if there exists $w \in W\left(G, A_{\mathfrak{p}}\right)$ such that $w \cdot \chi=\chi^{\prime}$.

Proof. Follows from [Kn01, VII.4] with a slight modification to the category $\mathcal{S} \mathcal{A} \mathcal{F}(G)$.

This implies the following:

Corollary 3.3. Let $\operatorname{Ind}_{B}^{G}(\chi)$ and $\operatorname{Ind}_{B}^{G}\left(\chi^{\prime}\right)$ be irreducible unitary principal series representations of $G$. Then they are isomorphic if and only if there exists $w \in W\left(G, A_{\mathfrak{p}}\right)$ such that $w \cdot \chi=\chi^{\prime}$.

In Appendix A.2 we prove the following corollary:

Corollary 3.4. Let $\pi_{1}, \pi_{2} \in \mathcal{S} \mathcal{A F}_{\text {Irr }}(G)$ where $\pi_{1}$ is the unique quotient of $\operatorname{Ind}_{B}^{G}\left(\chi_{1}\right)$ and $\pi_{2}$ is the unique quotient of $\operatorname{Ind}_{B}^{G}\left(\chi_{2}\right)$. Then $\pi_{1} \simeq \pi_{2}$ if and only if there exists $w \in W\left(G, A_{\mathfrak{p}}\right)$ such that $w \cdot \chi_{1}=\chi_{2}$.

Theorem 3.5 and Corollary 3.6 are needed for Section 6:

Theorem 3.5. If $\pi$ is the unique irreducible quotient of $\operatorname{Ind}_{B}^{G}(\chi)$ then:

1) $\tilde{\pi}$ is the unique irreducible quotient of $\operatorname{Ind}_{B}^{G}\left(w_{0} \cdot\left(\chi^{-1}\right)\right)$, where $w_{0} \in W(G, T)$ is the longest element in the Weyl group.

2) Let $\theta: G \longrightarrow G$ be an involution and assume that $T$ is $\theta$-stable. Then $\pi^{\theta}$ is the unique irreducible quotient of $\operatorname{Ind}_{B}^{G}\left(w^{\prime} \cdot \theta(\chi)\right)$ for some $w^{\prime} \in W(G, T)$ such that $w^{\prime} \cdot \theta(\chi)$ is dominant.

Proof. 1) The non degenerate pairing between $\operatorname{Ind}_{B}^{G}\left(\chi^{-1}\right)$ and $\operatorname{Ind}_{B}^{G}(\chi)$ by $\langle f, h\rangle:=\int_{B \backslash G} f \cdot h$ induces an isomorphism $\widetilde{\operatorname{Ind}_{B}^{G}(\chi)} \simeq \operatorname{Ind}_{B}^{G}\left(\chi^{-1}\right)$. As $\pi$ is the unique irreducible quotient of $\operatorname{Ind}_{B}^{G}(\chi)$, $\tilde{\pi}$ is the unique irreducible subrepresentation of $\left.\operatorname{Ind}_{B}^{G}\left(\chi^{-1}\right)=\operatorname{Ind}_{w_{0} \cdot B^{-}}^{G}\left(\chi^{-1}\right)\right)$, where $B^{-}$is the opposite Borel to $B$. By [Kn01, VIII.15, Theorem 8.54], $\widetilde{\pi}$ is the unique quotient of $\left.\operatorname{Ind}_{w_{0} . B}^{G}\left(\chi^{-1}\right)\right) \simeq$ $\operatorname{Ind}_{B}^{G}\left(w_{0} \cdot\left(\chi^{-1}\right)\right)$. As $\chi^{-1}$ is anti-dominant we have that $w_{0} \cdot\left(\chi^{-1}\right)$ is dominant.

2) Notice that $\theta \circ \operatorname{Ind}_{B}^{G}(\chi)$ is naturally isomorphic to $\operatorname{Ind}_{\theta(B)}^{G}(\theta(\chi))$ by $f \longrightarrow f^{\theta}$, where $f^{\theta}(g):=$ $f(\theta(g))$. This implies that $\pi^{\theta}$ is the unique irreducible quotient of $\left.\operatorname{Ind}_{\theta(B)}^{G}(\theta(\chi))\right)$. Notice that $\theta(B)$ is a Borel subgroup of $G$ that contains $\theta(T)=T$ and as $W(G, T)$ act transitively on the Borel subgroups of $G$ that contains $T$, there exists $w^{\prime} \in W(G, T)$ such that $w^{\prime} \cdot B=\theta(B)$. Thus $\pi^{\theta}$ is the unique irreducible quotient of $\left.\operatorname{Ind}_{B}^{G}\left(w^{\prime} \cdot \theta(\chi)\right)\right)$ and clearly $w^{\prime} \cdot \theta(\chi)$ is dominant.

Corollary 3.6. Assume that there exists $w \in W(G, T)$ such that $\chi^{-1}=w \cdot(\theta(\chi))$ and $w \circ \theta=$ $\theta \circ w^{-1}$. Then $\widetilde{\pi}^{\theta} \simeq \pi$. 
Proof. If $\chi^{-1}=w \cdot \theta(\chi)=\theta \cdot w^{-1}(\chi)$ then $\theta\left(\chi^{-1}\right)=w^{-1} \cdot \chi$. By Theorem 3.5, there exists $w^{\prime} \in$ $W(G, T)$ such that $\widetilde{\pi}^{\theta}$ is the unique irreducible quotient of $\operatorname{Ind}_{B}^{G}\left(w^{\prime} \cdot \theta\left(\chi^{-1}\right)\right)$. By Corollary 3.4, $\tilde{\pi}^{\theta} \simeq \pi$ as $w^{\prime} \cdot \theta\left(\chi^{-1}\right)=w^{\prime} w^{-1} \cdot \chi$.

We now use Theorem 3.1 to translate our question into the language of distributions. The quotient $\operatorname{map} \operatorname{Ind}_{B}^{G}(\chi) \longrightarrow \pi$ induces an injection $\left(\pi^{*}\right)^{H} \hookrightarrow\left(\operatorname{Ind}_{B}^{G}(\chi)^{*}\right)^{H}$ where $\chi$ is the Langlands parameter of $\pi$ from Theorem 3.1. Thus, it is enough to study $H$-distinguished representations of the form $\sigma=\operatorname{Ind}_{B}^{G} \chi$. Since $B \backslash G$ is compact, it follows from (e.g. [GSS15, Lemma 2.9]) that

$$
\sigma^{*}=\left(\left(C^{\infty}(G)^{B, \chi^{-1} \delta_{B}^{-1 / 2}}\right)^{*}\right)^{H} \simeq \mathcal{S}^{*}(G)^{B \times H, \chi \cdot \delta_{B}^{-1 / 2} \times 1} .
$$

Thus, together with Proposition 2.3, we obtain the following corollary:

Corollary 3.7. We have that

$$
\operatorname{dim}_{\mathbb{C}}\left(\pi^{*}\right)^{H} \leq \operatorname{dim}_{\mathbb{C}} \mathcal{S}^{*}(G / H)^{B, \chi \cdot \delta_{B}^{-1 / 2}} .
$$

This proves Step 1 (see Introduction). We now move towards Step 2, but before we prove it, we need to understand the geometry of $B$-orbits on $G / H$. This brings us to the next section.

\section{ON THE GEOMETRY OF $B$-ORBits ON $G / H$}

The study of the geometry of the minimal parabolic orbits on a symmetric space $G / H$ is well developed. The case of complex symmetric spaces was studied by Springer [Sp85] and the generalization for any local field was done in [HW93]. In this section we use results mostly from [HW93], as we are interested in real symmetric spaces. In order to apply the results in [HW93] to our setting we need to make some modifications:

(1) [HW93] studies the orbit decomposition of $\underline{B}(\mathbb{R})$ on $\underline{G}(\mathbb{R}) / \underline{H}(\mathbb{R})$, where $\underline{H}$ is an (Zarisky) open subgroup of $\underline{G}^{\theta}$. We need to generalize to the case when $H$ is an open subgroup (in the Hausdorff topology) of $\underline{G}^{\theta}(\mathbb{R})$.

(2) We want to show that there exists a stratification of $G / H$ by $B$-orbits.

Let $\underline{G}$ be a connected reductive algebraic group defined over $\mathbb{R}, \theta$ be an $\mathbb{R}$-involution of $\underline{G}, \underline{G}^{\theta}$ be the $\theta$-fixed points of $\underline{G}$ and $\underline{P}$ be a minimal parabolic subgroup. Let $\underline{U}=R_{u}(\underline{P})$ be the unipotent radical of $\underline{P}$. We recall the following known result:

Proposition 4.1. [HW93, Lemma 2.4] Every minimal parabolic $\mathbb{R}$-subgroup $\underline{P}$ of $\underline{G}$ contains a $\theta$-stable maximal $\mathbb{R}$-split torus $\underline{A}$ of $\underline{P}$, unique up to conjugation by an element of $\left(\underline{G}^{\theta} \cap \underline{U}\right)(\mathbb{R})$.

Let $\underline{A}$ be a $\theta$-stable maximal $\mathbb{R}$-split torus of $\underline{P}$. Given $g, x \in \underline{G}$, let $\rho$ be the twisted action associated to $\theta$, given by $\rho(g, x)=g x \theta(g)^{-1}$. Denote $\underline{Q}=\left\{g^{-1} \theta(g) \mid g \in \underline{G}\right\}, \underline{Q}^{\prime}=\{g \in G \mid \theta(g)=$ $\left.g^{-1}\right\}$ and note that $\underline{Q}$ and $\underline{Q}^{\prime}$ are invariant under $\rho$. Define a morphism $\tau: \underline{G} \rightarrow \underline{G}$ by $\tau(x)=$ $x \theta\left(x^{-1}\right)$. We have the following facts (see [HW93, Section 6]): 
* $\underline{Q}=\tau(\underline{G})$ is a connected closed subvariety of $\underline{G}$.

* $\underline{Q}$ is a finite union of twisted $G$-orbits, and each orbit is closed. In particular, $\underline{Q}$ is a connected component in $\underline{Q}^{\prime}$.

Define $Q=\tau(\underline{G}(\mathbb{R}))=\tau(G), Q^{\prime}=\underline{Q^{\prime}}(\mathbb{R})$ and notice that $\tau$ induces an isomorphism $\widetilde{\tau}: G / G^{\theta} \simeq Q$. Write $U=\underline{U}(\mathbb{R}), P=\underline{P}(\mathbb{R}), A=\underline{A}(\mathbb{R})$ and $N=N_{G}(A)$.

Proposition 4.2. [HW93, Proposition 6.6] If $g \in Q^{\prime}$, then there exists $x \in U$ such that $x g \theta(x)^{-1} \in$ $N$. Therefore, twisted $P$ orbits on $Q^{\prime}$ are represented by $n \in N \cap Q^{\prime}$.

Consider $\tau^{-1}(N)=\left\{g \in G \mid g^{-1} \theta(g) \in N\right\}$. The group $G^{\theta} \times C_{G}(A)$ acts on $\tau^{-1}(N)$ by $(x, y) . z=$ $x z y^{-1}$. Let $V$ denote the set of orbits of $G^{\theta} \times C_{G}(A)$ in $\tau^{-1}(N)$. We identify $V$ with a fixed set of representatives of the orbits in $\tau^{-1}(N)$.

Proposition 4.3. [HW93, Proposition 6.8]G is the disjoint union of the double cosets PvG $G^{\theta}$, $v \in V$.

Corollary 4.4. $\widetilde{\tau}: G / G^{\theta} \longrightarrow Q$ induces a bijection between $P$ - orbits on $G / G^{\theta}$ under the left action with $P$-orbits in $Q$ under the twisted action $\rho$. The double cosets $P x G^{\theta}$ are represented by elements $x \in \tau^{-1}(N)$, and the $P$ orbits in $Q$ are represented by elements $n \in N \cap Q$.

Proof. $\widetilde{\tau}$ is an isomorphism, so the bijection between $P$-orbits is clear. As the twisted $P$-orbits in $Q$ are represented by $n \in N$, the double cosets $P x G^{\theta}$ are represented by $x \in \tau^{-1}(N)$.

Proposition 4.5. [HW93, Proposition 6.15] $Q^{\prime}$ and $Q$ has only finitely many twisted P-orbits.

Combining the above results, we have the following corollary:

Corollary 4.6. $Q^{\prime}$ and $Q$ has finitely many twisted P-orbits, each is of the form P.n for $n \in N$ such that $\theta(n)=n^{-1}$.

The following Theorem 4.7 and Corollary 4.8 are used in Section 5, and they are essential for applying the tools from the Section 2 to the space $\mathcal{S}^{*}(G / H)^{B, \chi \cdot \delta_{B}^{-1 / 2}}$.

Theorem 4.7. There exists a stratification $Q=\bigcup_{k=1}^{m} P\left(n_{k}\right)$ by $P$-orbits, each contains some $n_{k} \in N_{G}(T) \cap Q$.

Proof. The following observations shows that it is enough to show that $Q^{\prime}$ has a such a stratification.

* By the argument above, we have that $Q^{\prime}$ decomposes into a finite number of $P$-orbits, and hence also to a finite number of $G$-orbits. This implies that also $\underline{Q}(\mathbb{R})$ has finite number of $G$ orbits.

* The map $\tau: \underline{G} \longrightarrow \underline{Q}$ is a submersion at $e$ [HW93, Lemma 6.13], hence $\tau(\underline{G}(\mathbb{R}))=Q$ is open in $\underline{Q}(\mathbb{R})$. Note that $\tau: \underline{G}(\mathbb{R}) \longrightarrow \underline{Q}(\mathbb{R})$ is the orbit map of $e \in \underline{Q}(\mathbb{R})$. In a similar argument one can show that each $G$-orbit in $\underline{Q}(\mathbb{R})$ is open, and since there are finite such orbits, they are also closed. Thus $Q$ is open and closed in $\underline{Q}(\mathbb{R})$. 
* Since $\underline{Q}(\mathbb{R})$ is open and closed in $Q^{\prime}$, we have that $Q$ is open and closed in $Q^{\prime}$ and hence a stratification on $Q^{\prime}$ restrict to a stratification on $Q$.

In order to show that $Q^{\prime}$ has such a stratification we use the following steps:

* By [HW93, Proposition 6.15], $Q^{\prime}$ has finitely many twisted $\underline{P}$-orbits. By induction on the number of these orbits, and the fact that any $\underline{G}$-variety has a (Zarisky) closed orbit, it is easy to see that $\underline{Q}^{\prime}$ has a stratification $\underline{Q}^{\prime}=\bigcup_{k=1}^{t} O_{\underline{P}}^{k}$ of $\underline{P}$-orbits $O_{\underline{P}}^{k}$.

* Write $Q^{\prime}=\bigcup_{k=1}^{t}\left(O_{\underline{P}}^{k} \cap Q^{\prime}\right)$. It is a known fact, that for each $\underline{P}$-orbit $O_{\underline{P}}^{k}$, we have that $O_{\underline{P}}^{k} \cap Q^{\prime}$ is a finite union of $P$-orbits $O_{j}, O_{\underline{P}}^{k} \cap Q^{\prime}=\bigsqcup_{j=1}^{m_{k}} O_{j}$, where each $O_{j}$ is open and closed in $O_{\underline{P}}^{k} \cap Q^{\prime}$. This gives the required stratification of $Q^{\prime}$.

Corollary 4.8. Let $G=\underline{G}(\mathbb{R})$ be a complex connected reductive group, $H$ be an open subgroup of $G^{\theta}=\underline{G}^{\theta}(\mathbb{R})$, and $B=\underline{B}(\mathbb{R})$ a Borel subgroup of $G$. Then there exists a finite stratification of $G / H$ by $B$-orbits.

Proof. Let $\pi: G / H \longrightarrow G / G^{\theta}$ be the natural quotient map and note that $\pi$ is a cover map, with fibers of size $\left|G^{\theta}: H\right|$. Let $G / G^{\theta}=\bigsqcup_{i=1}^{m} X_{i}$ be a $B$-stratification of $G / G^{\theta}$ and assume that $X_{i}$ is the $B$-orbit of $\left\{x_{i} G^{\theta}\right\} \in G / G^{\theta}$. Write $G^{\theta}=\bigsqcup h_{i} H$, where $h_{i}$ are representatives of the coset space $G^{\theta} / H$. Then $\pi^{-1}\left(X_{i}\right)$ is a $B$-invariant set that contains at most $\left|G^{\theta}: H\right| B$-orbits (the orbits of $\left.\pi^{-1}\left(x_{i} G^{\theta}\right)\right)$. Consider the open orbit $B\left(\left\{x_{1} G^{\theta}\right\}\right)$, and notice that $\pi^{-1}\left(B\left(\left\{x_{1} G^{\theta}\right\}\right)\right)$ is a $B$-invariant open set, and since $\pi$ is a local diffeomoerphism, it follows that each $B$-orbit in $\pi^{-1}\left(B\left(\left\{x_{1} G^{\theta}\right\}\right)\right)$ is open in $G / H$. By induction, we can now apply the same argument for the spaces $(G / H)-\pi^{-1}\left(X_{1}\right)$ and $\bigsqcup_{i=2}^{m} X_{i}$ and get a stratification of $G / H$ by $B$-orbits as required.

\section{SOME REDUCTIONS USING TOOLS FROM THE THEORY OF DISTRIBUTIONS}

In this section we assume the setting of Theorem 1.3 and we use the notation from Section 4 .

Lemma 5.1. There exists a Borel subgroup $B$, a $\theta$-stable maximal torus $T$ and a $\theta$-stable maximal $\mathbb{R}$-split torus $A$, such that $A \subseteq T \subseteq B$.

Proof. By Proposition 4.1 we can find a $\theta$-stable maximal $\mathbb{R}$-split torus $A$. As $G$ is complex, $C_{G}(A)=T$ a maximal torus and it is $\theta$-stable. Indeed, let $t \in C_{G}(A)$, then:

$$
t a t^{-1}=a \Longrightarrow \theta\left(t a t^{-1}\right)=\theta(a) \Longrightarrow \theta(t) \theta(a) \theta(t)^{-1}=\theta(a),
$$

so $\theta(t) \in C_{G}(A)$ and $T=C_{G}(A)$ is $\theta$-stable. We finish by choosing some Borel $B$ that contains $T$.

We now fix such $A \subseteq T \subseteq B$. Recall that for any $n \in N_{G}(T)$ such that $\theta(n)=n^{-1}$ we denote by $\theta_{n}$ the involution of $G$ defined by $\theta_{n}(g)=n \theta(g) n^{-1}$ and by $T^{\theta_{n}}$ (resp. $B^{\theta_{n}}$ ) the fixed points subgroup of $T$ (resp. $B$ ) under $\theta_{n}$. Let $Q, Q^{\prime}, \tau$ and $\widetilde{\tau}$ as in Section 4 . The goal in this section is to prove the following theorem: 
Theorem 5.2. If $\mathcal{S}^{*}(G / H)^{B, \chi \cdot \delta_{B}^{-1 / 2}} \neq 0$ then there exists $n \in N_{G}(T) \cap Q$, such that:

$$
\left.\operatorname{dim}_{\mathbb{C}}\left(\operatorname{Sym}\left(N_{B(n), n}^{Q^{\prime}}\right) \otimes_{\mathbb{R}} \mathbb{C}\right)^{T^{\theta_{n}}, \chi}\right)>0,
$$

where $B(n)$ is the $B$-orbit of $n \in Q^{\prime} \cap N_{G}(T)$ under the twisted action.

Recall that $\widetilde{\tau}: G / G^{\theta} \stackrel{\simeq}{\longrightarrow} Q$ is an isomorphism of $B$-spaces, where $B$ acts on $G / G^{\theta}$ by the left regular action and on $Q$ by the twisted action $\left(b . q=b q \theta(b)^{-1}\right)$. By Corollary 4.8 and Theorem 4.7, there exists a finite stratification of $G / H=\bigcup_{i=1}^{m} B\left(x_{i}\right)$ and $Q=\bigcup_{j=1}^{t} B\left(n_{j}\right)$ by $B$-orbits, where $m=|B \backslash G / H|, t=\left|B \backslash G / G^{\theta}\right|$ and $n_{j} \in N_{G}(T)$ for any $j$. Let $\pi: G / H \longrightarrow G / G^{\theta}$ be the natural quotient map. WLOG, we may assume for any $i \in\{1 \ldots m\}$ that $\widetilde{\tau} \circ \pi\left(x_{i}\right)=n_{j_{i}}$ for some $j_{i} \in\{1 \ldots t\}$. The following corollary follows from Proposition 2.2:

Corollary 5.3. We have:

$$
\operatorname{dim}_{\mathbb{C}}\left(\mathcal{S}^{*}(G / H)^{B, \chi \delta_{B}^{-1 / 2}}\right) \leq \sum_{i=1}^{m} \sum_{k=0}^{\infty} \operatorname{dim}_{\mathbb{C}}\left(\mathcal{S}^{*}\left(B\left(x_{i}\right), \operatorname{Sym}^{\mathrm{k}}\left(C N_{B\left(x_{i}\right), x_{i}}^{G / H}\right)\right)^{B, \chi \delta_{B}^{-1 / 2}}\right) .
$$

We now prove Theorem 5.2:

Proof. By Corollary 2.6 we deduce that:

$$
0<\operatorname{dim}_{\mathbb{C}}\left(\mathcal{S}^{*}(G / H)^{B, \chi \delta_{B}^{-1 / 2}}\right) \leq \sum_{i=1}^{m} \sum_{k=0}^{\infty} \operatorname{dim}_{\mathbb{C}}\left(\left(\operatorname{Sym}^{\mathrm{k}}\left(N_{B\left(x_{i}\right), x_{i}}^{G / H}\right) \otimes_{\mathbb{R}} \mathbb{C}\right)^{B_{x_{i}}, \chi \delta_{B_{x_{i}}}^{-1} \delta_{B}^{1 / 2}}\right)
$$

Since $\pi: G / H \longrightarrow G / G^{\theta}$ is a cover map, it is a local isomorphism of $B$-spaces. Therefore $\widetilde{\tau} \circ \pi$ is a local isomorphism of $B$-spaces as well, and for each $i$ we have:

$$
N_{B\left(x_{i}\right), x_{i}}^{G / H} \simeq N_{B\left(\pi\left(x_{i}\right)\right), \pi\left(x_{i}\right)}^{G / G^{\theta}} \simeq N_{B\left(n_{j_{i}}\right), n_{j_{i}}}^{Q} \simeq N_{B\left(n_{j_{i}}\right), n_{j_{i}}}^{Q^{\prime}}
$$

as $B$-representations, where the last equality follows from the fact that $Q$ is open in $Q^{\prime}$. We deduce that:

$$
0<\operatorname{dim}_{\mathbb{C}}\left(\mathcal{S}^{*}(G / H)^{B, \chi \delta_{B}^{-1 / 2}}\right) \leq \sum_{i=1}^{m} \sum_{k=0}^{\infty} \operatorname{dim}_{\mathbb{C}}\left(\left(\operatorname{Sym}^{\mathrm{k}}\left(N_{B\left(n_{j_{i}}\right), n_{j_{i}}}^{Q^{\prime}}\right) \otimes_{\mathbb{R}} \mathbb{C}\right)^{B^{\theta_{n_{j_{i}}}}, \chi^{\delta_{B}{ }^{-1}{ }_{\theta_{j_{i}}}} \delta_{B}^{1 / 2}}\right) .
$$

Notice that $B^{\theta_{n_{j_{i}}}}=\left\{b \in B \mid \theta_{n_{j_{i}}}(b)=n_{j_{i}} \theta(b) n_{j_{i}}^{-1}=b\right\}=B_{n_{j_{i}}}$. Hence, there exists $n \in N_{G}(T) \cap Q$ such that $\operatorname{dim}_{\mathbb{C}}\left(\left(\operatorname{Sym}^{\mathrm{k}}\left(N_{B(n), n}^{Q^{\prime}}\right) \otimes_{\mathbb{R}} \mathbb{C}\right)^{B^{\theta_{n}}, \chi \delta_{B^{\theta}}^{-1} \delta_{B}^{1 / 2}}\right)>0$. In particular,

$$
\operatorname{dim}_{\mathbb{C}}\left(\left(\operatorname{Sym}^{\mathrm{k}}\left(N_{B(n), n}^{Q^{\prime}}\right) \otimes_{\mathbb{R}} \mathbb{C}\right)^{T^{\theta_{n}}, \chi \delta_{B^{\theta_{n}}}^{-1} \delta_{B}^{1 / 2}}\right)>0 .
$$

As $\theta$ satisfies property $\star$, we have that $\left.\delta_{B}^{1 / 2}\right|_{B^{\theta_{n}}}=\delta_{B^{\theta_{n}}}$ and we get the desired result.

We can now combine Step 1 and Step 2 as follows: assume that $\pi \in \mathcal{S A F}_{\text {Irr }}(G)$ is $H$-distinguished. Step 1 showed that for any Borel $B, \mathcal{S}^{*}(G / H)^{B, \chi \cdot \delta_{B}^{-1 / 2}} \neq 0$ where $\chi$ is dominant with respect to the choice of $B$. By Lemma 5.1 we can find $A \subseteq T \subseteq B$ such that $T$ and $A$ are $\theta$-stable. Now we 
can use Step 2 and deduce the existence of $n \in N_{G}(T) \cap Q$ such that:

$$
\left.\operatorname{dim}\left(\operatorname{Sym}\left(N_{B(n), n}^{Q^{\prime}}\right) \otimes_{\mathbb{R}} \mathbb{C}\right)^{T^{\theta_{n}}, \chi}\right)>0 .
$$

\section{Proof of the main theorem- Theorem 1.3}

In this section we assume the same setting as in Section 5 and prove Theorem 1.3. Let $G$ be a complex connected reductive group, and $\mathfrak{g}$ be its Lie algebra, $K$ be a maximal compact subgroup of $G$ with Lie algebra $\mathfrak{k}, \tau$ the Cartan involution fixing $K$, and $\mathfrak{p}$ the -1 eigenspace of $\tau$. Let $\mathfrak{a}_{\mathfrak{p}}$ be a maximal abelian subspace of $\mathfrak{p}$, with the corresponding analytic subgroup $A_{\mathfrak{p}}$.

Proposition 6.1. We can choose $A_{\mathfrak{p}} \subseteq A \subseteq T \subseteq B$, where $A_{\mathfrak{p}}, A$ and $T$ are $\theta$-stable.

Proof. By Lemma 5.1 we can choose $A \subseteq T \subseteq B$ such that $T$ and $A$ are $\theta$-stable. As $G$ is a complex group, we can choose some Cartan involution $\tau$ such that $\mathfrak{a}=\operatorname{Lie}(A)$ is a maximal abelian subalgebra of $\mathfrak{p}$, i.e $\mathfrak{a}=\mathfrak{a}_{\mathfrak{p}}$ and $A_{\mathfrak{p}}=\exp \left(\mathfrak{a}_{\mathfrak{p}}\right) \simeq\left(\mathbb{R}_{>0}\right)^{n}$ is the connected component of $\{e\}$ in $A \simeq\left(\mathbb{R}^{\times}\right)^{n}$. As $\theta$ is a an algebraic morphism, $\theta\left(A_{\mathfrak{p}}\right)$ is connected and contains $\{e\}$ thus $A_{\mathfrak{p}}$ is $\theta$-stable as well.

Using the above proposition, we fix such $A_{\mathfrak{p}} \subseteq A \subseteq T \subseteq B$. Let $\pi \in \mathcal{S A F}_{\text {Irr }}(G)$ and let $\chi$ be its Langlands parameter such that $\pi$ is the unique quotient of $\operatorname{Ind}_{B}^{G}(\chi)$. Recall that $\theta_{n}(g)=n \theta(g) n^{-1}$ and $Q^{\prime}=\left\{g \in G \mid \theta(g)=g^{-1}\right\}$. Using Step 1 and Step 2, the next theorem clearly implies Theorem 1.3a):

Theorem 6.2. If there exists $n \in N_{G}(T) \cap Q^{\prime}$ such that $\left.\operatorname{dim}_{\mathbb{C}}\left(\operatorname{Sym}\left(N_{B(n), n}^{Q^{\prime}}\right) \otimes_{\mathbb{R}} \mathbb{C}\right)^{T^{\theta_{n}}, \chi}\right)>0$, then:

and $\tilde{\pi} \simeq \theta \circ \pi$.

$$
\left.\operatorname{dim}_{\mathbb{C}}\left(\operatorname{Sym}\left(N_{B(n), n}^{Q^{\prime}}\right) \otimes_{\mathbb{R}} \mathbb{C}\right)^{T^{\theta_{n}}, \chi}\right)=1
$$

Corollary 6.3. Theorem 6.2 also implies Theorem 1.3b).

Proof. By Corollary 3.7 and the discussion in Section 5

$$
\begin{aligned}
\operatorname{dim}_{\mathbb{C}}\left(\pi^{*}\right)^{H} & \leq \operatorname{dim}_{\mathbb{C}} \mathcal{S}^{*}(G / H)^{B, \chi \cdot \delta_{B}^{-1 / 2}} \\
& \leq \sum_{i=1}^{|B \backslash G / H|} \sum_{k=0}^{\infty} \operatorname{dim}_{\mathbb{C}}\left(\left(\operatorname{Sym}^{\mathrm{k}}\left(N_{B\left(n_{j_{i}}\right), n_{j_{i}}}^{Q^{\prime}}\right) \otimes_{\mathbb{R}} \mathbb{C}\right)^{T^{\theta_{n_{i}}}, \chi}\right)
\end{aligned}
$$

where $n_{j_{i}}$ is a representative of the $B$-orbit $B\left(n_{j_{i}}\right)$ in $Q$. By Theorem 6.2 it follows that $\operatorname{dim}_{\mathbb{C}}\left(\pi^{*}\right)^{H} \leq$ $|B \backslash G / H|$ and also that $\tilde{\pi} \simeq \theta \circ \pi$ as required.

The proof of Theorem 6.2 is divided to several parts: in the first part we calculate $N_{B(n), n}^{Q^{\prime}}$ using the Lie algebra of $G$ by some direct calculations. In the second part we decompose $\operatorname{Sym}\left(N_{B(n), n}^{Q^{\prime}}\right) \otimes_{R} \mathbb{C}$ to eigenspaces as a complex representation of $T^{\theta_{n}}$ and then use the $\left(T^{\theta_{n}}, \chi\right)$ equivariance to show 
that $\chi^{-1}=w \circ \theta(\chi)$, where $w=n T \in N_{G}(T) / T$ is the element in the Weyl group $W$ that corresponds to $n$. From here we deduce that $\tilde{\pi} \simeq \theta \circ \pi$.

6.1. Calculation of $N_{B(n), n}^{Q^{\prime}}$. Recall the notations from Section 4. Define a map $\gamma: G \longrightarrow G$ by $\gamma(g)=g \theta(g)$ and let $a: B \longrightarrow B(n)$ be the orbit map $b \longmapsto b n \theta\left(b^{-1}\right)$. Notice that $T_{n} Q^{\prime}=$ $\operatorname{Ker}(d \gamma)_{n}, T_{n} B(n)=\operatorname{Im}(d a)_{e}$ and $N_{B(n), n}^{Q^{\prime}}=T_{n} Q^{\prime} / T_{n} B(n)=\operatorname{Ker}(d \gamma)_{n} / \operatorname{Im}(d a)_{e}$. Denote by $\mu: G \times G \longrightarrow G$ the group multiplication, by $L_{n}, R_{n}: G \longrightarrow G$ the left and right multiplication by $n$, and by $\left(d L_{n}\right)_{k}$ and $\left(d R_{n}\right)_{k}$, their differentials at a point $k \in G$. For the convenience of the reader, we will drop the notation ()$_{e}$ when taking differential at $e$.

Lemma 6.4. We have that:

$$
\begin{aligned}
T_{n} Q^{\prime} & =\operatorname{Ker}(d \gamma)_{n}=\left\{X \in T_{n}(G) \mid\left(d R_{n^{-1}}\right)_{n}(X)=-d \theta \circ\left(d L_{n^{-1}}\right)_{n}(X)\right\} . \\
T_{n} B(n) & \left.=\operatorname{Im}(d a)=\left\{d R_{n}(Y)-d L_{n} \circ d \theta(Y)\right) \mid Y \in T_{e}(B)\right\} .
\end{aligned}
$$

Proof. $\gamma=\mu \circ(\mathrm{Id}, \theta)$. We can write $\gamma=\mu \circ\left(R_{n^{-1}} \circ \mathrm{Id}, L_{n} \circ \theta\right)$ and get:

$$
\begin{aligned}
(d \gamma)_{n}(X) & =d \mu_{(e, e)} \circ\left(\left(d R_{n^{-1}}\right)_{n} \circ(d \mathrm{Id})_{n},\left(d L_{n}\right)_{n^{-1}} \circ(d \theta)_{n}\right)(X) \\
& =\left(d R_{n^{-1}}\right)_{n}(X)+\left(d L_{n}\right)_{n^{-1}} \circ(d \theta)_{n}(X) .
\end{aligned}
$$

As $n \in Q^{\prime}$, it satisfies $\theta(n)=n^{-1}$ so $n^{-1} \theta\left(n^{-1} g\right)=\theta(g)$ and this implies that

$$
(d \theta)_{n}(X)=d L_{n^{-1}} \circ d \theta \circ\left(d L_{n^{-1}}\right)_{n}(X) .
$$

Altogether:

$$
\begin{aligned}
(d \gamma)_{n}(X) & =\left(d R_{n^{-1}}\right)_{n}(X)+\left(d L_{n}\right)_{n^{-1}} \circ(d \theta)_{n}(X) \\
& =\left(d R_{n^{-1}}\right)_{n}(X)+\left(d L_{n}\right)_{n^{-1}} \circ d L_{n^{-1}} \circ d \theta \circ\left(d L_{n^{-1}}\right)_{n}(X) \\
& =\left(d R_{n^{-1}}\right)_{n}(X)+d \theta \circ\left(d L_{n^{-1}}\right)_{n}(X) .
\end{aligned}
$$

This provides a description of

$$
\operatorname{Ker}(d \gamma)_{n}=\left\{X \in T_{n}(G) \mid\left(d R_{n^{-1}}\right)_{n}(X)=-d \theta \circ\left(d L_{n^{-1}}\right)_{n}(X)\right\} .
$$

Now we would like to describe $T_{n}(B(n))=\operatorname{Im}(d a)$. Notice that $a(g)=g n \theta\left(g^{-1}\right)=n \cdot\left(n^{-1} g n\right)$. $\theta\left(g^{-1}\right)$, so:

$$
d a(X)=d L_{n} \circ d \mu \circ\left(\operatorname{Ad}_{n^{-1}}, d(\theta \circ i)\right)(X)=d R_{n}(X)-d L_{n} \circ d \theta(X),
$$

where $i: G \longrightarrow G$ is the inversion map. This finishes the Lemma.

The goal now is to give a description of $T_{n} Q^{\prime}$ and $T_{n} B(n)$ using the root space decomposition of $G$ as a reductive group. If we consider $G$ as a complex group then we can write $\mathfrak{g}=\mathfrak{g}_{0} \oplus$ $\bigoplus_{\widetilde{\alpha} \in \Sigma(G, T)} \mathfrak{g}_{\tilde{\alpha}}$, where $\mathfrak{g}_{0}=\operatorname{Lie}(T), \Sigma(G, T)$ is the corresponding root system and each eigenspace $\mathfrak{g}_{\tilde{\alpha}}$ is one dimensional over $\mathbb{C}$. If we consider $G$ as a real group then we can again write $\mathfrak{g}=$ $\mathfrak{g}_{0} \oplus \bigoplus_{\alpha \in \Sigma\left(G, A_{\mathfrak{p}}\right)} \mathfrak{g}_{\alpha}$. As $A_{\mathfrak{p}}$ is $\theta$-stable we have an action of $\theta$ on the restricted root system 
$\Sigma\left(G, A_{\mathfrak{p}}\right)$. We write $\Sigma^{+}\left(G, A_{\mathfrak{p}}\right), \Sigma^{-}\left(G, A_{\mathfrak{p}}\right)$ for the positive and negative roots (with respect to the choice of the Borel subgroup $B)$.

Lemma 6.5. Each eigenspace $\mathfrak{g}_{\alpha}$, for $\alpha \in \Sigma\left(G, A_{\mathfrak{p}}\right)$, is two dimensional over $\mathbb{R}$ and $\mathfrak{g}_{\alpha}=\mathfrak{g}_{\tilde{\alpha}}$ for some $\widetilde{\alpha} \in \Sigma(G, T)$ such that $\left.\widetilde{\alpha}\right|_{A_{\mathfrak{p}}}=\alpha$. Moreover, there is a unique such $\widetilde{\alpha}$ and $d \theta\left(\mathfrak{g}_{\widetilde{\alpha}}\right)=\mathfrak{g}_{\theta(\alpha)}$.

Proof. We have an isomorphism $\phi: T \simeq\left(\mathbb{C}^{\times}\right)^{n}$ where $\left.\phi\right|_{A}: A \stackrel{\simeq}{\longrightarrow}\left(\mathbb{R}^{\times}\right)^{n}$ and $\left.\phi\right|_{A_{\mathfrak{p}}}: A_{\mathfrak{p}} \stackrel{\simeq}{\longrightarrow}$ $\left(\mathbb{R}_{>0}\right)^{n}$. Under this identification, we can write any root $\widetilde{\alpha} \in \Sigma(G, T)$ as $\widetilde{\alpha}(t)=\widetilde{\alpha}\left(t_{1}, \ldots, t_{n}\right)=$ $\prod\left|t_{i}\right|^{n_{i}} \cdot\left(\frac{t_{i}}{\left|t_{i}\right|}\right)^{n_{i}}$, where $n_{i} \in \mathbb{Z}$. If we restrict $\widetilde{\alpha}$ to $A_{\mathfrak{p}}$ we can write $\left.\widetilde{\alpha}\right|_{A_{\mathfrak{p}}}\left(t_{1}, \ldots, t_{n}\right)=\prod t_{i}^{n_{i}}$. Therefore $\alpha=\left.\widetilde{\alpha}\right|_{A_{\mathfrak{p}}}=\left.\widetilde{\beta}\right|_{A_{\mathfrak{p}}}=\beta$ if and only if $\widetilde{\alpha}=\widetilde{\beta}$. This implies that $d \theta\left(\mathfrak{g}_{\widetilde{\alpha}}\right)=\mathfrak{g}_{\widetilde{\theta(\alpha)}}$.

We now calculate $T_{n} Q^{\prime}$ and $T_{n} B(n)$ in terms of the restricted root decomposition $\mathfrak{g}=\mathfrak{g}_{0} \oplus$ $\bigoplus_{\alpha \in \Sigma\left(G, A_{\mathfrak{p}}\right)} \mathfrak{g}_{\alpha}$. Denote $\psi_{n}:=d \theta \circ \operatorname{Ad}_{n}, \mathfrak{b}=\mathfrak{g}_{0} \oplus \bigoplus_{\alpha \in \Sigma^{+}\left(G, A_{\mathfrak{p}}\right)} \mathfrak{g}_{\alpha}$. Let $w \in W\left(G, A_{\mathfrak{p}}\right)$ be the Weyl element corresponding to $n \in N_{G}(T)=N_{G}\left(A_{\mathfrak{p}}\right)$.

Theorem 6.6. Under the identification of $T_{n} Q^{\prime}$ and $T_{n} B(n)$ as sub-algebras of $\mathfrak{g}$ by applying $d L_{n^{-1}}$, we have:

$$
\begin{aligned}
T_{n}\left(Q^{\prime}\right) & \simeq\left\{X \in \mathfrak{g} \mid d \theta \circ \operatorname{Ad}_{n}(X)=-X\right\}=\left(\mathfrak{g}_{0} \oplus \bigoplus_{\alpha \in \Sigma} \mathfrak{g}_{\alpha}\right)_{\psi_{n},-1}=\left(\mathfrak{g}_{0} \oplus \bigoplus_{\alpha \in \Sigma} \mathfrak{g}_{w^{-1}(\alpha)}\right)_{\psi_{n},-1}, \\
T_{n} B(n) & \simeq\left\{\operatorname{Ad}_{n^{-1}}(Y)-d \theta(Y) \mid Y \in \mathfrak{b}\right\}=\left(\mathfrak{g}_{0} \oplus \underset{\alpha \text { or } w \circ \theta(\alpha) \in \Sigma^{+}}{\bigoplus_{w}(\alpha)}\right)_{\psi_{n},-1},
\end{aligned}
$$

where ()$_{\psi_{n},-1}$ denote the -1 eigenspace of $\psi_{n}$.

Proof. The first statement is clear by using Lemma 6.4 and identify $T_{n} Q^{\prime}$ with $T_{e}(G)$ by applying $\left(d L_{n^{-1}}\right)_{n}$. For the second statement, observe that $\left\{\operatorname{Ad}_{n^{-1}}(Y)-d \theta(Y) \mid Y \in \mathfrak{g}_{\alpha}\right\}$ is the -1 eigenspace of $\psi_{n}$ restricted to the space $\mathfrak{g}_{w^{-1}(\alpha)} \oplus \mathfrak{g}_{\theta(\alpha)}$ (or the space $\mathfrak{g}_{w^{-1}(\alpha)}$ if $\left.\psi_{n}\left(\mathfrak{g}_{w^{-1}(\alpha)}\right)=\mathfrak{g}_{w^{-1}(\alpha)}\right)$. Indeed, $\left\{\operatorname{Ad}_{n^{-1}}(Y)-d \theta(Y) \mid Y \in \mathfrak{g}_{\alpha}\right\}$ and $\left\{\operatorname{Ad}_{n^{-1}}(Y)+d \theta(Y) \mid Y \in \mathfrak{g}_{\alpha}\right\}$ are contained in the -1 and +1 eigenspaces of $\left.\psi_{n}\right|_{\mathfrak{g}_{w^{-1}(\alpha)}} \oplus \mathfrak{g}_{\theta(\alpha)}$, respectively, and since they span $\mathfrak{g}_{w^{-1}(\alpha)} \oplus \mathfrak{g}_{\theta(\alpha)}$ it follows that they are indeed the \pm 1 eigenspaces. In addition, notice that

$$
\left\{\operatorname{Ad}_{n^{-1}}(Y)-d \theta(Y) \mid Y \in \mathfrak{g}_{\alpha}\right\}=\left\{\operatorname{Ad}_{n^{-1}}(Y)-d \theta(Y) \mid Y \in \mathfrak{g}_{w \circ \theta(\alpha)}\right\}
$$

are both the -1 eigenspace of $\psi_{n}$ restricted to the space $\mathfrak{g}_{w^{-1}(\alpha)} \oplus \mathfrak{g}_{\theta(\alpha)}$. The same argument can be applied to $\mathfrak{g}_{0}$. This finishes the second statement.

Corollary 6.7. Under the identification of $T_{n} Q^{\prime}$ and $T_{n} B(n)$ as sub-algebras of $\mathfrak{g}$ by applying $d L_{n^{-1}}$, we obtain:

$$
N_{B(n), n}^{Q^{\prime}}=T_{n} Q^{\prime} / T_{n} B(n) \simeq\left(\bigoplus_{\alpha \in \Sigma^{-} \cap w \circ \theta\left(\Sigma^{-}\right)} \mathfrak{g}_{w^{-1}(\alpha)}\right)_{\psi_{n},-1}
$$


6.2. Proof of Theorem 6.2. We are now interested in $\operatorname{Sym}\left(N_{B(n), n}^{Q^{\prime}}\right) \otimes_{\mathbb{R}} \mathbb{C}$ as a complex representation of $T^{\theta_{n}}$ under the twisted involution $\rho_{t}(g):=\operatorname{tg} \theta(t)^{-1}$. Notice that $T^{\theta_{n}}$ acts on $N_{B(n), n}^{Q^{\prime}}$ via the differential of the action at the point $n$, i.e via $\left(d \rho_{t}\right)_{n}$, and this induces an action on $\operatorname{Sym}\left(N_{B(n), n}^{Q^{\prime}}\right) \otimes_{\mathbb{R}} \mathbb{C}$.

Lemma 6.8. Consider $N_{B(n), n}^{Q^{\prime}} \simeq\left(\bigoplus_{\alpha \in \Sigma^{-} \cap w \circ \theta\left(\Sigma^{-}\right)} \mathfrak{g}_{w^{-1}(\alpha)}\right)_{\psi_{n,-1}}$ as a representation of $T^{\theta_{n}}$ (with respect to $\left.\left(d \rho_{t}\right)_{n}\right)$. Then each $\left(\mathfrak{g}_{w^{-1}(\alpha)} \oplus \mathfrak{g}_{\theta(\alpha)}\right)_{\psi_{n},-1}$ is an $\widetilde{\alpha}$-eigenspace, for $\widetilde{\alpha} \in \Sigma(G, T)$ (i.e, the unique root $\widetilde{\alpha}$ such that $\left.\widetilde{\alpha}\right|_{A_{\mathfrak{p}}}=\alpha$, see Lemma 6.5).

Proof. Observe that $\rho_{t}(g)=\operatorname{tg} \theta(t)^{-1}=t g t^{-1} \cdot t \theta(t)^{-1}$ and that $t^{-1} n \theta(t) n^{-1}=e \Longleftrightarrow n^{-1} t n=\theta(t)$ for any $t \in T^{\theta_{n}}$. In other words, $\operatorname{Ad}_{\theta(t)}=\operatorname{Ad}_{n^{-1} t n} . T^{\theta_{n}}$ acts on $T_{n}\left(Q^{\prime}\right)$ via $\left(d \rho_{t}\right)_{n}$ and recall that we have identified $T_{n}\left(Q^{\prime}\right)$ with a subalgebra of $\mathfrak{g}$ via $\left(d L_{n^{-1}}\right)_{n}(6.1)$. Therefore, in order to understand the action of $T^{\theta_{n}}$ on $\left(\mathfrak{g}_{w^{-1}(\alpha)} \oplus \mathfrak{g}_{\theta(\alpha)}\right)_{\psi_{n},-1}$ we need to identify it with $T_{n}\left(Q^{\prime}\right)$ via $d L_{n}$, apply the twisted involution and move back to $\mathfrak{g}$ via $\left(d L_{n^{-1}}\right)_{n}$. Define $\rho_{t}^{\prime}:=L_{n^{-1}} \circ \rho_{t} \circ L_{n}$ to be induced twisted action on $T_{e}(G) \simeq \mathfrak{g}$. Notice that:

$$
\rho_{t}^{\prime}(g)=n^{-1} \rho_{t}(n g)=n^{-1} \operatorname{tng} \theta(t)^{-1}=n^{-1} \operatorname{tngn}^{-1} t^{-1} n .
$$

Therefore:

$$
d \rho_{t}^{\prime}(X):=d L_{n^{-1}} \circ d \rho_{t} \circ d L_{n}(X)=\operatorname{Ad}_{n^{-1}} \circ \operatorname{Ad}_{t} \circ \operatorname{Ad}_{n}(X)=\operatorname{Ad}_{n^{-1} t n}(X)=\operatorname{Ad}_{\theta(t)}(X),
$$

and as a consequence:

$$
d \rho_{t}^{\prime}\left(\mathfrak{g}_{w^{-1}(\alpha)}\right)=w^{-1}(\widetilde{\alpha})(\theta(t)) \mathfrak{g}_{w^{-1}(\alpha)}=\theta \circ w^{-1} \circ \widetilde{\alpha}(t) \mathfrak{g}_{w^{-1}(\alpha)}=\widetilde{\alpha}(t) \mathfrak{g}_{w^{-1}(\alpha)}
$$

and also $d \rho_{t}^{\prime}\left(\mathfrak{g}_{\theta(\alpha)}\right)=\widetilde{\alpha}(t) \mathfrak{g}_{\theta(\alpha)}$. This implies that $\left(\mathfrak{g}_{w^{-1}(\alpha)} \oplus \mathfrak{g}_{\theta(\alpha)}\right)_{\psi_{n},-1}$ is an eigenspace of $T^{\theta_{n}}$ under twisted involution with the same character $\widetilde{\alpha}$.

Corollary 6.9. As a complex representation of $T^{\theta_{n}}$, we have that:

$$
N_{B(n), n}^{Q^{\prime}} \otimes_{R} \mathbb{C} \simeq \bigoplus_{\alpha \in S} V_{\widetilde{\alpha}},
$$

where $S$ is some multiset containing only negative roots $\alpha \in \Sigma\left(G, A_{\mathfrak{p}}\right)$ such that $w \circ \theta(\alpha)$ is also negative, and $V_{\widetilde{\alpha}}$ is a one dimensional representation of $T^{\theta_{n}}$ corresponding to a character $\widetilde{\alpha}$.

Proof. The space $N_{B(n), n}^{Q^{\prime}} \otimes_{\mathbb{R}} \mathbb{C}$ is a complex representation of $T^{\theta_{n}}$ so we can decompose it to one-dimensional $\mathbb{C}$-eigenspaces. By Lemma $6.8,\left(\mathfrak{g}_{w^{-1}(\alpha)} \oplus \mathfrak{g}_{\theta(\alpha)}\right)_{\psi_{n},-1}$ is an $\widetilde{\alpha}$-eigenspace, thus:

* If $w \circ \theta(\alpha) \neq \alpha$ we have that $\mathbb{C} \otimes_{\mathbb{R}}\left(\mathfrak{g}_{w^{-1}(\alpha)} \oplus \mathfrak{g}_{\theta(\alpha)}\right)_{\psi_{n},-1} \simeq V_{\widetilde{\alpha}} \oplus V_{\widetilde{\alpha}}$.

* If $w \circ \theta(\alpha)=\alpha$ and $\left.\psi_{n}\right|_{\mathfrak{g}_{\theta(\alpha)}}=-\left.\mathrm{Id}\right|_{\mathfrak{g}_{\theta(\alpha)}}$ we have that $\mathbb{C} \otimes_{\mathbb{R}}\left(\mathfrak{g}_{\theta(\alpha)}\right)_{\psi_{n},-1} \simeq \mathfrak{g}_{\theta(\alpha)} \oplus \mathfrak{g}_{\theta(\alpha)} \simeq V_{\widetilde{\alpha}} \oplus V_{\widetilde{\alpha}}$.

* If $w \circ \theta(\alpha)=\alpha$ and $\left.\psi_{n}\right|_{\mathfrak{g}_{\theta(\alpha)}}=\left.\operatorname{Id}\right|_{\mathfrak{g}_{\theta(\alpha)}}$ then $\mathbb{C} \otimes_{\mathbb{R}}\left(\mathfrak{g}_{\theta(\alpha)}\right)_{\psi_{n},-1}$ is trivial.

This implies that $N_{B(n), n}^{Q^{\prime}} \otimes_{\mathbb{R}} \mathbb{C} \simeq \bigoplus_{\alpha \in S} V_{\widetilde{\alpha}}$ when $S$ is a multiset that contains roots $\alpha$ (with possible repetitions), such that both $\alpha$ and $w \circ \theta(\alpha)$ are negative. 
Example. Consider the case of $G=\mathrm{GL}_{\mathrm{n}}(\mathbb{C}), G^{\theta}=\mathrm{GL}_{\mathrm{n}}(\mathbb{R})$. In this case the $B$-orbits in $Q^{\prime}$ are represented by the set of involutive Weyl elements, that is, $w \in W\left(G, A_{\mathfrak{p}}\right)$ such that $w^{2}=\mathrm{Id}$. In addition $W\left(G, A_{\mathfrak{p}}\right)=S_{n^{-}}$the permutation group of $n$ elements. Under this identification, we have an action of $W \simeq S_{n}$ on rows and columns. We also have a decomposition $\mathfrak{g l}_{n}=\mathfrak{g}_{0} \oplus \bigoplus \mathfrak{g}_{\alpha_{i j}}$ where $\mathfrak{g}_{\alpha_{i j}}$ is the complex space spanned by matrix $E_{i j}$ (that has 1 in the $i j$ th entry and 0 in all the other entries). Calculating $N_{B(w), w}^{Q^{\prime}}$ yields:

$$
N_{B(w), w}^{Q^{\prime}} \otimes_{R} \mathbb{C} \simeq \bigoplus_{\left\{(i, j) \in I_{w}\right\}} V_{\widetilde{\alpha_{i j}}}
$$

where $I_{w}=\{(i, j): i>j, w(i)>w(j)\}$. Under the choice of the upper triangular matrices as the Borel subgroup, $I_{w}$ corresponds to the set of negative roots $\left\{\alpha_{i j}\right\}$ such that $\alpha_{w(i), w(j)}=w\left(\alpha_{i j}\right)=$ $w \circ \theta\left(\alpha_{i j}\right)$ is negative. We see that this example agrees with the result in 6.9.

Corollary 6.10. We have the following isomorphism of representations of $T^{\theta_{n}}$ :

$$
\operatorname{Sym}\left(N_{B(n), n}^{Q^{\prime}}\right) \otimes_{R} \mathbb{C} \simeq \bigoplus_{\varphi: S \longrightarrow \mathbb{Z}_{\geq 0}} V_{\alpha^{\varphi}},
$$

where $\varphi: S \longrightarrow \mathbb{Z}_{\geq 0}$ is some function, and $V_{\alpha^{\varphi}}$ is a one dimensional representation of $T^{\theta_{n}}$ that corresponds to a character $\alpha^{\varphi}(t)=\prod_{\alpha_{j} \in S}\left(\widetilde{\alpha_{j}}(t)\right)^{\varphi\left(\alpha_{j}\right)}$ for the multiset $S$ as defined in Corollary 6.9.

Proof. Choose a basis $\left\{Y_{1}, \ldots, Y_{m}\right\}$ of $N_{B(n), n}^{Q^{\prime}} \otimes_{\mathbb{R}} \mathbb{C}$ where each $Y_{i}$ is in some $V_{\widetilde{\alpha_{i}}}$. Recall that the action of $T^{\theta_{n}}$ on $N_{B(n), n}^{Q^{\prime}}$ is via $\operatorname{Ad}_{\theta(t)}$. We can now describe the action of $T^{\theta_{n}}$ on the space

$$
\operatorname{Sym}\left(N_{B(n), n}^{Q^{\prime}}\right) \otimes_{R} \mathbb{C} \simeq \operatorname{Sym}\left(N_{B(n), n}^{Q^{\prime}} \otimes_{R} \mathbb{C}\right)=\bigoplus_{k=0}^{\infty} \operatorname{Sym}^{\mathrm{k}}\left(N_{B(n), n}^{Q^{\prime}} \otimes_{R} \mathbb{C}\right) .
$$

$\operatorname{Sym}^{\mathrm{k}}\left(N_{B(n), n}^{Q^{\prime}} \otimes_{R} \mathbb{C}\right)$ has basis of tensors $\bigotimes_{j=1}^{k} Y_{i_{j}}$ (modulo the symmetrization relations). Then for $t \in T^{\theta_{n}}$ :

$$
t .\left(\bigotimes_{j=1}^{k} Y_{i_{j}}\right)=\bigotimes_{j=1}^{k} \operatorname{Ad}_{\theta(t)}\left(Y_{i_{j}}\right)=\widetilde{\alpha_{i_{1}}}(t) \cdot \ldots \cdot \widetilde{\alpha_{i_{k}}}(t) \cdot \bigotimes_{j=1}^{k} Y_{i_{j}}=\alpha^{\varphi}(t) \cdot \bigotimes_{j=1}^{k} Y_{i_{j}},
$$

where $\varphi\left(\alpha_{j}\right)$ is the number of appearances of $j$ in $\left\{i_{1}, \ldots, i_{k}\right\}$.

Corollary 6.11. If $\operatorname{dim}_{\mathbb{C}}\left(\operatorname{Sym}\left(N_{B(n), n}^{Q^{\prime}} \otimes_{R} \mathbb{C}\right)\right)^{T^{\theta_{n}}, \chi} \neq 0$ then there exists $\varphi: S \longrightarrow \mathbb{Z}_{\geq 0}$ such that $\alpha^{\varphi}(t)=\chi(t)$ for any $t \in T^{\theta_{n}}$.

Proof. Each vector $X \in\left(\operatorname{Sym}\left(N_{B(n), n}^{Q^{\prime}} \otimes_{R} \mathbb{C}\right)\right)^{T^{\theta_{n}}, \chi}=\left(\bigoplus_{\varphi: S \longrightarrow \mathbb{Z}_{\geq 0}} V_{\alpha \varphi}\right)^{T^{\theta_{n}}, \chi}$ is of the form $X=\sum_{i=1}^{n} X_{\varphi_{i}}$ where $X_{\varphi_{i}} \in V_{\alpha \varphi_{i}}$. This implies that for any $i, X_{\varphi_{i}}$ is also $\left(T^{\theta_{n}}, \chi\right)$ equivariant. This implies that $\varphi_{i}(t)=\chi(t)$ for any $t \in T^{\theta_{n}}$.

Lemma 6.12. For any $n \in N_{G}(T) \cap Q^{\prime}$, we have that $\left\{n \theta(t) n^{-1} t \mid t \in T\right\} \subseteq T^{\theta_{n}}$. 
Proof. Recall that $T^{\theta_{n}}=\left\{t \in T \mid \operatorname{tn} \theta(t)^{-1}=n\right\}=\left\{t \in T \mid \theta(t)=n^{-1} t n\right\}$ and notice that:

$$
\theta\left(n \theta(t) n^{-1} t\right)=n^{-1} \operatorname{tn} \theta(t)=\theta(t) n^{-1} t n=n^{-1}\left(n \theta(t) n^{-1} t\right) n .
$$

Corollary 6.13. If $\left(\operatorname{Sym}\left(N_{B(n), n}^{Q^{\prime}} \otimes_{R} \mathbb{C}\right)\right)^{T^{\theta_{n}}, \chi} \neq 0$ then there exists $\varphi: S \longrightarrow \mathbb{Z}_{\geq 0}$ such that:

$$
\alpha^{\varphi}(t) \cdot w \circ \theta\left(\alpha^{\varphi}\right)(t)=\chi(t) \cdot w \circ \theta(\chi)(t),
$$

for any $t \in T$.

Proof. By Corollary $6.11,\left.\alpha^{\varphi}\right|_{T^{\theta_{n}}}=\left.\chi\right|_{T^{\theta_{n}}}$ and by Lemma 6.12 we can deduce that for every $t \in T$, $\alpha^{\varphi}\left(n \theta(t) n^{-1} t\right)=\chi\left(n \theta(t) n^{-1} t\right)$. Therefore:

$$
\alpha^{\varphi}\left(n \theta(t) n^{-1} t\right)=\alpha^{\varphi}(t) \cdot w \circ \theta\left(\alpha^{\varphi}\right)(t)=\chi(t) \cdot w \circ \theta(\chi)(t)=\chi\left(n \theta(t) n^{-1} t\right) .
$$

By Corollary 3.6, in order to prove that $\widetilde{\pi} \simeq \pi^{\theta}$ it is enough to show that $\chi^{-1}=w \circ \theta(\chi)$. Notice that by Langlands' classification, we have that $\chi$ is dominant while $\left.\alpha^{\varphi}\right|_{A_{\mathfrak{p}}}$ and $\left.w \circ \theta\left(\alpha^{\varphi}\right)\right|_{A_{\mathfrak{p}}}$ are negative. This leads us to our main result.

Theorem 6.14. If $\left(\operatorname{Sym}\left(N_{B(n), n}^{Q^{\prime}} \otimes_{R} \mathbb{C}\right)\right)^{T^{\theta_{n}}, \chi} \neq 0$, then:

1) $\operatorname{dim}_{\mathbb{C}}\left(\operatorname{Sym}\left(N_{B(n), n}^{Q^{\prime}} \otimes_{R} \mathbb{C}\right)\right)^{T^{\theta_{n}}, \chi}=1$.

2) $\chi^{-1}=w \circ \theta(\chi)$ and hence $\tilde{\pi}^{\theta} \simeq \pi$.

Proof. By Corollary 6.13, $\alpha^{\varphi}(t) \cdot w \circ \theta\left(\alpha^{\varphi}\right)(t)=\chi(t) \cdot w \circ \theta(\chi)(t)$ for any $t \in T$. For any root $\alpha_{j}$ with multiple instances $\alpha_{j_{1}}, \ldots, \alpha_{j_{m}}$ in $S$, we may define $n_{j}=\sum_{i=1}^{m} \varphi\left(\alpha_{j_{i}}\right)$. We can change $S$ to be a set by deleting all multiple instances in it. By doing this, we can write $\alpha^{\varphi}(t)=\prod_{\alpha_{j} \in S}\left(\widetilde{\alpha_{j}}(t)\right)^{n_{j}}$, when $S$ is now a set (without repetitions) of roots $\left\{\alpha_{j}\right\}$ such that both $\alpha_{j}, w \circ \theta\left(\alpha_{j}\right)$ are negative. We now restrict to $A_{\mathfrak{p}}$ and take the differentials of the characters to get the following equation:

$$
\sum_{j \in S} n_{j} \cdot\left(\alpha_{j}+w \circ \theta\left(\alpha_{j}\right)\right)=d\left(\left.\chi\right|_{A_{\mathfrak{p}}}\right)+d\left(\left.w \circ \theta(\chi)\right|_{A_{\mathfrak{p}}}\right) .
$$

Recall that both $\theta$ and $w$ preserve $\Sigma\left(G, A_{\mathfrak{p}}\right)$ and hence preserve the inner product between the roots (see [Hum72, Lemma, p.43]), that is:

$$
\langle w \circ \theta(\alpha), w \circ \theta(\beta)\rangle=\langle w(\alpha), w(\beta)\rangle=\langle\theta(\alpha), \theta(\beta)\rangle=\langle\alpha, \beta\rangle .
$$

Thus, we have for any $\alpha \in \Sigma\left(G, A_{\mathfrak{p}}\right)$ :

$$
\left\langle d\left(\left.\chi\right|_{A_{\mathfrak{p}}}\right), w \circ \theta(\alpha)\right\rangle=\left\langle d\left(\left.w \circ \theta(\chi)\right|_{A_{\mathfrak{p}}}\right), w \circ \theta \circ w \circ \theta(\alpha)\right\rangle=\left\langle d\left(\left.w \circ \theta(\chi)\right|_{A_{\mathfrak{p}}}\right), \alpha\right\rangle .
$$


We now claim that $\sum_{j \in S} n_{j} \cdot\left(\alpha_{j}+w \circ \theta\left(\alpha_{j}\right)\right)=0$. Assume that there exists some $k$ such that $n_{k} \neq 0$. By taking the inner product of $\sum_{j \in S} n_{j} \cdot\left(\alpha_{j}+w \circ \theta\left(\alpha_{j}\right)\right)$ with itself we get on one hand:

$$
\left\langle\sum_{j \in S} n_{j} \cdot\left(\alpha_{j}+w \circ \theta\left(\alpha_{j}\right)\right), \sum_{j \in S} n_{j} \cdot\left(\alpha_{j}+w \circ \theta\left(\alpha_{j}\right)\right)\right\rangle>0 .
$$

But on the other hand, as $\chi$ is dominant and $\alpha_{j}, w \circ \theta\left(\alpha_{j}\right) \in \Sigma\left(G, A_{\mathfrak{p}}\right)^{-}$for any $\alpha_{j} \in S$ it holds that:

$$
\begin{gathered}
\left\langle d\left(\left.\chi\right|_{A_{\mathfrak{p}}}\right)+d\left(\left.w \circ \theta(\chi)\right|_{A_{\mathfrak{p}}}\right), \sum_{j \in S} n_{j} \cdot\left(\alpha_{j}+w \circ \theta\left(\alpha_{j}\right)\right)\right\rangle \\
=\left\langle d\left(\left.\chi\right|_{A_{\mathfrak{p}}}\right), \sum_{j \in S} n_{j} \cdot\left(\alpha_{j}+w \circ \theta\left(\alpha_{j}\right)\right)\right\rangle+\left\langle d\left(\left.w \circ \theta(\chi)\right|_{A_{\mathfrak{p}}}\right), \sum_{j \in S} n_{j} \cdot\left(\alpha_{j}+w \circ \theta\left(\alpha_{j}\right)\right)\right\rangle \leq 0 .
\end{gathered}
$$

We got a contradiction, hence $n_{k}=0$ for any $k$ and $\alpha^{\varphi}\left(n \theta(t) n^{-1} t\right)=1$. This implies that:

1) $\chi^{-1}=w \circ \theta(\chi)$.

2) $\left(\operatorname{Sym}\left(N_{B(n), n}^{Q^{\prime}} \otimes_{R} \mathbb{C}\right)\right)^{T^{\theta_{n}}, \chi}=\left(\operatorname{Sym}^{0}\left(N_{B(n), n}^{Q^{\prime}} \otimes_{R} \mathbb{C}\right)\right)^{T^{\theta_{n}}, \chi}$ is one dimensional.

\section{Appendix A. Langlands' Classification}

Let $G$ be a real reductive group with Lie algebra $\mathfrak{g}$ and $K$ a maximal compact subgroup. Define $\mathcal{H C}$ to be the category of finitely generated, admissible $(\mathfrak{g}, K)$-modules (see [KT00, Lecture 5$]$ ). Casselman and Wallach showed that there is an equivalence of categories $\mathcal{S} \mathcal{A F}(G) \simeq \mathcal{H C}(\mathfrak{g}, K)$. The functor in one direction is $E \longmapsto E^{K-\text { finite }}$, where $E^{K-\text { finite }}$ is the subspace of $K$-finite vectors. The functor in the other direction is: $V \longmapsto V^{\infty}$ where $V^{\infty}:=\pi(\mathcal{S}(G)) V, \mathcal{S}(G)$ is the Schwartz algebra of rapidly decreasing functions on $G$, and $\pi(\mathcal{S}(G)) V$ stands for the vector space spanned by $\pi(f) v$ for $f \in \mathcal{S}(G), v \in V$.

The Langlands classification gives a classification of all equivalence classes of irreducible admissible Hilbert representations of $G$ up to infinitesimal equivalence, where two irreducible Hilbert representations $\left(\pi_{1}, \mathcal{H}_{1}\right)$ and $\left(\pi_{2}, \mathcal{H}_{2}\right)$ are infinitesimal equivalent if $\mathcal{H}_{1}^{K-\text { finite }} \simeq \mathcal{H}_{2}^{K-\text { finite }}$ as $(\mathfrak{g}, K)$-modules.

In this section we state Langlands' classification for Hilbert representations and use it to prove the complex version (i.e, when $G$ is a complex reductive group, see Theorem 3.1) of the classification for Fréchet representations. Both theorems are known results, and in fact, the complex version was first proved by Zhelobenko and Naimark (see [ZN66]), and later it was generalized by Langlands.

For the following definitions and Theorem 3.1, assume that $\pi$ is a Hilbert representations of $G$. Moreover, to avoid confusion, we set $\mathrm{H}-\operatorname{Ind}_{P}^{G}$ to be the normalized induction in the category of Hilbert representations, and by $\operatorname{Ind}_{P}^{G}$ the normalized induction in the category $\mathcal{S} \mathcal{A F}(G)$.

Definition. 1) $\pi$ is called a discrete series representation if it is unitary, irreducible and it is infinitesimally equivalent to a direct summand of the right regular representation of $G$ on $L^{2}(G)$. 
2) $\pi$ is called a tempered representation of $G$, if $\pi$ is infinitesimally equivalent to a subrepresentation of an induced representation $H-\operatorname{Ind}_{M A N}^{G}(\sigma \otimes \lambda \otimes 1)$ for some standard parabolic subgroup $M A N \supseteq$ $M_{p} A_{p} N_{p}$, some discrete series representation $\sigma$ of $M$, and some imaginary character $\lambda$ of $A$, i.e of the form $\left(t_{1}, \ldots, t_{n}\right) \longmapsto \prod t_{i}^{\lambda_{i}}$ where the $\lambda_{i}$ are purely imaginary.

Theorem A.1. Let $G$ be a real connected reductive Lie group, with $\mathfrak{g}$ be its Lie algebra. Also let $K$ be a maximal compact subgroup of $G$ with Lie algebra $\mathfrak{k}, \tau$ the Cartan involution fixing $K$, and $\mathfrak{p}$ the -1 eigenspace of $\tau$. Let $\mathfrak{a}_{\mathfrak{p}}$ be a maximal abelian subspace of $\mathfrak{p}, \Sigma$ the root system of $\mathfrak{a}_{\mathfrak{p}}$ in $\mathfrak{g}, \triangle$ the set of simple roots of $\Sigma$, and $F \subseteq \triangle$. Furthermore, let $M_{\mathfrak{p}} A_{\mathfrak{p}} N_{\mathfrak{p}}$ be the corresponding minimal parabolic subgroup (see Appendix B.1).

Then the infinitesimal equivalence class of irreducible admissible Hilbert representations of $G$ are uniquely parameterized by triples $(F,[\sigma], \lambda)$, where:

* $Q_{F}$ is a standard parabolic subgroup of $G$ corresponding to $F$ with Langlands decomposition $Q_{F}=M_{F} A_{F} N_{F}$ containing $M_{\mathfrak{p}} A_{\mathfrak{p}} N_{\mathfrak{p}}$.

* $\sigma$ is an irreducible tempered representation of the semisimple Lie group $M$, where [] denotes equivalence class up to infinitesimal equivalence.

* The character $\lambda \in \mathfrak{a}_{F}^{*}$ satisfies $\operatorname{Re}\langle\alpha, \lambda\rangle>0$ for all simple roots $\alpha$ not in $F$.

More precisely, the triple $(F,[\sigma], \lambda)$ corresponds to the equivalence class of the unique irreducible quotient of $\mathrm{H}-\operatorname{Ind}_{M A N}^{G}(\sigma \otimes \lambda \otimes 1)$, that is, $\pi \simeq \mathrm{H}-\operatorname{Ind}_{M A N}^{G}(\sigma \otimes \lambda \otimes 1) / W$, where $W$ is the maximal subrepresentation of $\mathrm{H}-\operatorname{Ind}_{M A N}^{G}(\sigma \otimes \lambda \otimes 1)$.

A.1. Proof of Theorem 3.1. Before we prove Theorem 3.1 we present three facts and key Theorem A.2:

Fact. 1) (Harish-Chandra, 1966, see e.g. [KT00, p.71]) A linear connected reductive group has no discrete series unless $G$ has a compact Cartan subgroup.

2) Let $G$ be a complex reductive group and $\chi$ be a unitary character of $T=M_{\mathfrak{p}} A_{\mathfrak{p}}$. Then $\mathrm{H}-\operatorname{Ind}_{B}^{G} \chi$ is irreducible (follows from [Kn01, Corollary 14.60]).

3) (Double induction formula, [Kn01, VII.2-property 4]) Let $G$ a reductive group, $M A N$ be a parabolic subgroup of $G$ and let $M^{\prime} A^{\prime} N^{\prime}$ be a parabolic subgroup of $M$, so that $M^{\prime}\left(A^{\prime} A\right)\left(N^{\prime} N\right)$ is a parabolic subgroup of $G$. If $\sigma^{\prime}$ is a unitary representation of $M^{\prime}$ and $v^{\prime}$ and $v$ are characters of $\mathfrak{a}^{\prime}$ and $\mathfrak{a}$, respectively, then there is a canonical equivalence:

$\mathrm{H}-\operatorname{Ind}_{M A N}^{G}\left(\mathrm{H}-\operatorname{Ind}_{M^{\prime} A^{\prime} N^{\prime}}^{M}\left(\sigma^{\prime} \otimes \exp \left(v^{\prime}\right) \otimes 1\right) \otimes \exp (v) \otimes 1 \simeq \mathrm{H}-\operatorname{Ind}_{M^{\prime}\left(A^{\prime} A\right)\left(N^{\prime} N\right)}^{G}\left(\sigma^{\prime} \otimes \exp \left(v^{\prime}+v\right) \otimes 1\right)\right.$

Theorem A.2. Let $\pi$ be an irreducible, admissible, Hilbert representation of $G$ and fix some maximal torus $T$ and a Borel subgroup $B \supseteq T$. Then it is equivalent to the unique quotient of $H-\operatorname{Ind}_{B}^{G}(\chi)$, where $\chi$ is some dominant character of $T$, i.e $\operatorname{Re}\left\langle\left. d \chi\right|_{\mathfrak{a}_{\mathfrak{p}}}, \alpha\right\rangle \geq 0$. 
Proof. We choose $B=M_{\mathfrak{p}} A_{\mathfrak{p}} N_{\mathfrak{p}}$. By the Langlands classification, $\pi$ is equivalent to $\mathrm{H}-\operatorname{Ind}_{M A N}^{G}(\sigma \otimes$ $\lambda \otimes 1) / W$ where $Q=M A N \supseteq B=M_{\mathfrak{p}} A_{\mathfrak{p}} N_{\mathfrak{p}}$, and $W$ is the maximal proper subrepresentation. We have two possible cases:

1) If $M A N=M_{\mathfrak{p}} A_{\mathfrak{p}} N_{\mathfrak{p}}=B$ then $\pi$ is equivalent to the unique quotient of $\mathrm{H}-\operatorname{Ind}_{B}^{G}(\sigma \otimes \lambda \otimes 1)$, where $\lambda$ is a strongly dominant character (i.e $\operatorname{Re}\langle d \lambda, \alpha\rangle>0$ ) and $\sigma$ is an unitary representation of $M_{\mathfrak{p}}$. As $M_{\mathfrak{p}}$ is compact and abelian, $\sigma$ is a one dimensional unitary representation. As a consequence, $\chi:=\sigma \cdot \lambda$ is a character and $\left.\chi\right|_{A_{\mathfrak{p}}}=\lambda$ so it is dominant.

2) $B \varsubsetneqq Q=M A N \subseteq G$. Then $\pi$ is equivalent to $\pi^{\prime}=\mathrm{H}-\operatorname{Ind}_{M A N}^{G}(\sigma \otimes \lambda \otimes 1) / W$, where $\sigma$ is a tempered representation of $M$. By definition, $\sigma$ is equivalent to $\tau$, where $\tau \hookrightarrow \mathrm{H}-\operatorname{Ind}_{M^{\prime} A^{\prime} N^{\prime}}^{M}\left(\sigma^{\prime} \otimes\right.$ $\left.\lambda^{\prime} \otimes 1\right), \sigma^{\prime}$ is a discrete series representation of $M^{\prime}$ and $\lambda^{\prime}$ is an imaginary character of $A^{\prime}$. We now show that:

* $M^{\prime}$ has no discrete series unless $M^{\prime} A^{\prime} A N^{\prime}$ is a minimal parabolic of $M A$.

* $\mathrm{H}-\operatorname{Ind}_{M^{\prime} A^{\prime} N^{\prime}}^{M}\left(\sigma^{\prime} \otimes \lambda^{\prime} \otimes 1\right)$ is irreducible for $\sigma^{\prime}$ unitary and $\lambda^{\prime}$ unitary and purely imaginary.

$M A$ is a complex group with $M^{\prime} A A^{\prime} N$ a complex parabolic subgroup. From the structure theory of parabolic subgroups of complex reductive groups, it follows that $M^{\prime}$ has no compact Cartan subgroup unless $M^{\prime} A A^{\prime} N$ is a minimal parabolic subgroup of $M A$. For the second statement, we have an intertwining map: $\tau \hookrightarrow \mathrm{H}-\operatorname{Ind}_{M^{\prime} A^{\prime} N^{\prime}}^{M}\left(\sigma^{\prime} \otimes \lambda^{\prime} \otimes 1\right)$. As $A$ is central in $M A$, we can tensor $\tau$ with the trivial representation on $A$ to get an irreducible representation $\tau \otimes 1$ such that:

$$
\tau \otimes 1 \hookrightarrow\left(\mathrm{H}-\operatorname{Ind}_{M^{\prime} A^{\prime} N^{\prime}}^{M}\left(\sigma^{\prime} \otimes \lambda^{\prime} \otimes 1\right)\right) \otimes 1 \hookrightarrow \mathrm{H}-\operatorname{Ind}_{M^{\prime} A^{\prime} A N^{\prime}}^{M A}\left(\sigma^{\prime} \otimes\left(\lambda^{\prime} \otimes 1\right) \otimes 1\right) .
$$

But as $M A$ is complex and $M^{\prime} A^{\prime} A N^{\prime}$ is a minimal parabolic subgroup, i.e a Borel subgroup, it follows by Fact 2 that $\mathrm{H}-\operatorname{Ind}_{M^{\prime} A^{\prime} A N^{\prime}}^{M A}\left(\sigma^{\prime} \otimes\left(\lambda^{\prime} \otimes 1\right) \otimes 1\right)$ is irreducible and hence $\mathrm{H}-\operatorname{Ind}_{M^{\prime} A^{\prime} N^{\prime}}^{M}\left(\sigma^{\prime} \otimes\right.$ $\left.\lambda^{\prime} \otimes 1\right)=\tau$ is irreducible. By ([Kn96, Proposition 7.82]) we can find a minimal parabolic subgroup $M^{\prime} A^{\prime} N^{\prime}$ of $M$ such that $M^{\prime} A^{\prime} A N^{\prime} N=M_{\mathfrak{p}} A_{\mathfrak{p}} N_{\mathfrak{p}}=B$.

since $\sigma$ is equivalent to $\mathrm{H}-\operatorname{Ind}_{M^{\prime} A^{\prime} N^{\prime}}^{M}\left(\sigma^{\prime} \otimes \lambda^{\prime} \otimes 1\right)$, we can assume that $\pi^{\prime}$ is the unique quotient:

$$
\mathrm{H}-\operatorname{Ind}_{M A N}^{G}\left(\left(H-\operatorname{Ind}_{M^{\prime} A^{\prime} N^{\prime}}^{M}\left(\sigma^{\prime} \otimes \lambda^{\prime} \otimes 1\right)\right) \otimes \lambda \otimes 1\right) \longrightarrow \pi^{\prime} .
$$

Using the double induction formula (Fact 3) on the left side of the equation gives:

$$
\begin{aligned}
\mathrm{H}-\operatorname{Ind}_{B}^{G}(\chi) & \simeq \mathrm{H}-\operatorname{Ind}_{M^{\prime} A^{\prime} A N^{\prime} N}^{G}\left(\sigma^{\prime} \otimes \lambda^{\prime} \cdot \lambda \otimes 1\right) \\
& \simeq \mathrm{H}-\operatorname{Ind}_{M A N}^{G}\left(\left(\mathrm{H}-\operatorname{Ind}_{M^{\prime} A^{\prime} N^{\prime}}^{M}\left(\sigma^{\prime} \otimes \lambda^{\prime} \otimes 1\right)\right) \otimes \lambda \otimes 1\right) \longrightarrow \pi^{\prime},
\end{aligned}
$$

where $\sigma^{\prime} \lambda^{\prime}$ is a unitary character of $M A^{\prime}, \lambda^{\prime}$ is purely imaginary and $\chi=\sigma^{\prime} \lambda^{\prime} \lambda$. Notice that $\lambda$ satisfies $\operatorname{Re}\langle\alpha, \lambda\rangle>0$ for all simple roots $\alpha$ not in $F$ and $\operatorname{Re}\langle\alpha, \lambda\rangle=0$ for $\alpha \in F$. Therefore $\left.\chi\right|_{A_{p}}=\lambda^{\prime} \lambda$ satisfies $\operatorname{Re}\left\langle\left. d \chi\right|_{\mathfrak{a}_{\mathfrak{p}}}, \alpha\right\rangle \geq 0$ for any $\alpha \in \triangle$ and $\chi$ is a dominant character.

We now show that Theorem A.2 can be applied to Fréchet representations:

By a theorem of Harish-Chandra, any irreducible $V \in \mathcal{H C}$ admits a Hilbert globalization $\mathcal{H}$, such that $\mathcal{H}$ is an irreducible, admissible Hilbert representation and $\mathcal{H}^{K-\text { finite }} \simeq V$ as a $(\mathfrak{g}, K)$-module 
(see e.g. [BK14, Section 5.1]). Denote $\mathcal{H}_{\text {smooth }}$ to be the smooth part of $\mathcal{H}$ and notice that $\mathcal{H}_{\text {smooth }} \in \mathcal{S} \mathcal{A F}_{\text {Irr }}(G)$ is a Fréchet globalization of $V$. Assume we are given a representation $E \in \mathcal{S A F}_{\text {Irr }}(G)$ such that $V:=E^{K-\text { finite }}$. Then by the Casselman-Wallach globalization theorem we have that $\mathcal{H}_{\text {smooth }} \simeq E$.

We can now prove Theorem 3.1:

Theorem A.3. Let $(\pi, E) \in \mathcal{S A F}_{\text {Irr }}(G)$. Then $(\pi, E)$ is the unique irreducible quotient of $\operatorname{Ind}_{B}^{G}(\chi)$ for a dominant character $\chi$ of $T$.

Proof. Denote $V:=E^{K-\text { finite }}$ and let $\mathcal{H}$ its Hilbert globalization, such that $E \simeq \mathcal{H}_{\text {smooth }}$. By Theorem A.1, $\mathcal{H}$ is equivalent to $\mathrm{H}-\operatorname{Ind}_{B}^{G}(\chi) / W$, where $W$ is the maximal subrepresentation of $\mathrm{H}-$ $\operatorname{Ind}_{B}^{G}(\chi)$. This implies that $V \simeq \mathrm{H}-\operatorname{Ind}_{B}^{G}(\chi)^{K-\text { finite }} / W^{K-\text { finite }}$. Note that the $\operatorname{Ind}_{B}^{G}(\chi)^{K-\text { finite }}=$ $\mathrm{H}-\operatorname{Ind}_{B}^{G}(\chi)^{K-\text { finite }}$ so $\operatorname{Ind}_{B}^{G}(\chi)$ is a Fréchet globalization of $\mathrm{H}-\operatorname{Ind}_{B}^{G}(\chi)^{K-\text { finite }}$ and this implies that $(\pi, E)$ is the unique quotient of $\operatorname{Ind}_{B}^{G}(\chi)$ as required.

\section{A.2. Proof of Corollary 3.4.}

Corollary A.4. Let $\pi_{1}, \pi_{2} \in \mathcal{S} \mathcal{A} \mathcal{F}_{\text {Irr }}(G)$ where $\pi_{1}$ is the unique quotient of $\operatorname{Ind}_{B}^{G}\left(\chi_{1}\right)$ and $\pi_{2}$ is the unique quotient of $\operatorname{Ind}_{B}^{G}\left(\chi_{2}\right)$. Then $\pi_{1} \simeq \pi_{2}$ if and only if there exists $w \in W\left(G, A_{\mathfrak{p}}\right)$ such that $w \circ \chi_{1}=\chi_{2}$.

Proof. By Corollary 3.3 this is true for tempered representations. By Theorem A.2, $\pi_{1}$ is the

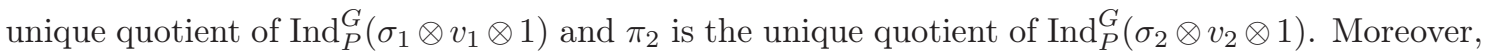
we can write $\sigma_{1}=\operatorname{Ind}_{M^{\prime} A^{\prime} N^{\prime}}^{M}\left(\sigma_{1}^{\prime} \otimes v_{1}^{\prime} \otimes 1\right)$ and $\sigma_{2}=\operatorname{Ind}_{M^{\prime} A^{\prime} N^{\prime}}^{M}\left(\sigma_{2}^{\prime} \otimes v_{2}^{\prime} \otimes 1\right)$ where $M^{\prime} A^{\prime} A N^{\prime} N=$ $M_{\mathfrak{p}} A_{\mathfrak{p}} N_{\mathfrak{p}}=B$, such that $\pi_{1}$ is the unique quotient of $\operatorname{Ind}_{B}^{G}\left(\sigma_{1}^{\prime} \otimes v_{1}^{\prime} \cdot v_{1} \otimes 1\right)$ and $\pi_{2}$ is the unique quotient of $\operatorname{Ind}_{B}^{G}\left(\sigma_{2}^{\prime} \otimes v_{2}^{\prime} \cdot v_{2} \otimes 1\right)$. Let $\chi_{1}=\sigma_{1}^{\prime} \otimes v_{1}^{\prime} \cdot v_{1}$ and $\chi_{2}=\sigma_{2}^{\prime} \otimes v_{2}^{\prime} \cdot v_{2}$.

Assume $\pi_{1} \simeq \pi_{2}$. By Theorem A.2, $\sigma_{1} \simeq \sigma_{2}$ and $v_{1}=v_{2}$. By Corollary 3.3, $\sigma_{1} \simeq \sigma_{2}$ if and only if there exists $w^{\prime} \in W\left(M, A^{\prime}\right)$ such that $w^{\prime} \circ\left(\sigma_{1}^{\prime} \otimes v_{1}^{\prime}\right)=\left(\sigma_{2}^{\prime} \otimes v_{2}^{\prime}\right)$. Let $n^{\prime} \in N_{M}\left(A^{\prime}\right)$ be an element that correspond to $w^{\prime} . M$ centralizes $A$ and therefore $n^{\prime}$ centralizes $A$. Thus $n^{\prime} \in N_{G}\left(A_{\mathfrak{p}}\right)$ and it corresponds to an element $w \in W\left(G, A_{\mathfrak{p}}\right)$ such that $w$ centralize $A$ and acts as $w^{\prime}$ on $A^{\prime}$. Therefore:

$$
w\left(\sigma_{1}^{\prime} \otimes v_{1}^{\prime} \cdot v_{1} \otimes 1\right)=w^{\prime} \sigma_{1}^{\prime} \otimes w^{\prime} v_{1}^{\prime} \cdot v_{1} \otimes 1=\sigma_{2}^{\prime} \otimes v_{2}^{\prime} \cdot v_{2} \otimes 1,
$$

which implies that $w \circ \chi_{1}=\chi_{2}$. For the other direction, assume $w \circ \chi_{1}=\chi_{2}$, that is $w \circ\left(\sigma_{1}^{\prime} \otimes\right.$ $\left.v_{1}^{\prime} \cdot v_{1} \otimes 1\right)=\sigma_{2}^{\prime} \otimes v_{2}^{\prime} \cdot v_{2} \otimes 1$. As both $v_{1}^{\prime} \cdot v_{1}$ and $v_{2}^{\prime} \cdot v_{2}$ are dominant with respect to positive roots $\alpha$ such that $\left.\alpha\right|_{A}$ is non zero, $w$ must act trivially on $A$. Therefore $w\left(\sigma_{1}^{\prime} \otimes v_{1}^{\prime} \cdot v_{1} \otimes 1\right)=$ $w^{\prime} \sigma_{1}^{\prime} \otimes w^{\prime} v_{1}^{\prime} \cdot v_{1} \otimes 1=\sigma_{2}^{\prime} \otimes v_{2}^{\prime} \cdot v_{2} \otimes 1$ so $v_{1}=v_{2}$ and by Corollary $3.3 \sigma_{1} \simeq \sigma_{2}$. By Theorem A.2, $\pi_{1} \simeq \pi_{2}$. 


\section{Appendix B. Some facts ABout the structure of ReAl Reductive Groups}

For the convenience of the reader, we summarize some of the structure theory of real reductive groups. This appendix is based on [KT00] and [Kn96].

Any real reductive algebraic group $G$ can be represented by a 4-tuple $(G, K, \theta, B()$,$) , where K$ is a maximal compact subgroup, $\theta$ is an involution of $\mathfrak{g}=\operatorname{Lie}(G)$, and $B($,$) is a non degenerate,$ $A d(G)$-invariant, $\theta$-invariant, bilinear form, such that:

- $\mathfrak{g}$ is a reductive Lie algebra and it decomposes into $\mathfrak{g}=\mathfrak{k} \oplus \mathfrak{p}$, where $\mathfrak{k}$ is the +1 eigenspace of $\theta$ (and the Lie algebra of $K$ ) and $\mathfrak{p}$ is the -1 eigenspace of $\theta$.

- $B($,$) is negative definite on \mathfrak{k}$ and positive definite on $\mathfrak{p}$, and $\mathfrak{k}$ and $\mathfrak{p}$ are orthogonal under $B$.

- We have a diffeomorphism $K \times \exp (\mathfrak{p}) \longrightarrow G$.

- Any automorphism $\operatorname{Ad}(g)$ is inner for $g \in G$.

We can define an inner product $\langle$,$\rangle on \mathfrak{g}$ by $\langle X, Y\rangle=-B(X, \theta(Y))$.

Fact. Relative to the inner product $\langle$,$\rangle on \mathfrak{g}$, it holds that $\left(\operatorname{ad}_{X}\right)^{*}=\operatorname{ad}_{X}$ for any $X \in \mathfrak{p}$.

Let $\mathfrak{a}_{\mathfrak{p}}$ be the maximal abelian subspace of $\mathfrak{p}$. By the above fact, $\left\{\operatorname{ad}_{X}\right\}_{X \in \mathfrak{a}_{\mathfrak{p}}}$ is a commuting family of symmetric operators on $\mathfrak{g}$ and hence it is simultaneously diagonalizable. This gives a decomposition $\mathfrak{g}=\mathfrak{g}_{0} \oplus \bigoplus_{\lambda \in \Sigma} \mathfrak{g}_{\lambda}$ where $\mathfrak{g}_{\lambda}:=\left\{X \in g \mid\left(\operatorname{ad}_{H}\right) X=\lambda(H) X\right.$ for all $\left.H \in \mathfrak{a}_{\mathfrak{p}}\right\}, \Sigma$ is the set of all $\lambda \in \mathfrak{a}_{\mathfrak{p}}^{*}$ such that $\mathfrak{g}_{\lambda}$ is non zero. Such $\lambda$ is called a restricted root. This decomposition is called restricted root space decomposition and it has some nice properties:

- $\left[\mathfrak{g}_{\mu}, \mathfrak{g}_{\lambda}\right] \subseteq \mathfrak{g}_{\mu+\lambda}$.

- $\mathfrak{g}_{\mu}$ and $\mathfrak{g}_{\lambda}$ are orthogonal with respect to $\langle$,$\rangle if \mu \neq \lambda$.

- $\mathfrak{g}_{0}=\mathfrak{m}_{\mathfrak{p}} \oplus \mathfrak{a}_{\mathfrak{p}}$ where $\mathfrak{m}_{\mathfrak{p}}=Z_{\mathfrak{k}}\left(\mathfrak{a}_{\mathfrak{p}}\right)$ is the centralizer of $\mathfrak{a}_{\mathfrak{p}}$ in $\mathfrak{k}$. This is an orthogonal sum.

We now introduce a lexicographic order on $\mathfrak{a}_{\mathfrak{p}}{ }^{*}$. We fix an ordered basis $\lambda_{1}, \ldots, \lambda_{l}$ of $\mathfrak{a}_{\mathfrak{p}}^{*}$ and define $\lambda=\sum_{i} c_{i} \lambda_{i}$ to be positive if $c_{1}=\ldots c_{k}=0$ and $c_{k+1}>0$ for some $0 \leq k<l$. We set $\lambda>\mu$ if $\lambda-\mu$ is positive and define $\Sigma^{+}$to be the set of positive roots in $\Sigma$ and $\mathfrak{n}_{\mathfrak{p}}=\bigoplus_{\lambda \in \Sigma^{+}} \mathfrak{g}_{\lambda}$. We have:

- $\mathfrak{g}=\mathfrak{k} \oplus \mathfrak{a}_{\mathfrak{p}} \oplus \mathfrak{n}_{\mathfrak{p}}, \mathfrak{a}_{\mathfrak{p}}$ is abelian, $\mathfrak{n}_{\mathfrak{p}}$ is nilpotent, $\mathfrak{a}_{\mathfrak{p}} \oplus \mathfrak{n}_{\mathfrak{p}}$ is solvable and $\left[\mathfrak{a}_{\mathfrak{p}} \oplus \mathfrak{n}_{\mathfrak{p}}, \mathfrak{a}_{\mathfrak{p}} \oplus \mathfrak{n}_{\mathfrak{p}}\right]=\mathfrak{n}_{\mathfrak{p}}$.

- There are analytic subgroups $A_{\mathfrak{p}}$ and $N_{\mathfrak{p}}$ with Lie algebras $\mathfrak{a}_{\mathfrak{p}}, \mathfrak{n}_{\mathfrak{p}}$ such that $A_{\mathfrak{p}}, N_{\mathfrak{p}}, A_{\mathfrak{p}} N_{\mathfrak{p}}$ are simply connected closed subgroups of $G$ and $G$ is diffeomorphic to $K \times A_{\mathfrak{p}} \times N_{\mathfrak{p}}$.

Remark. The inner product $\langle$,$\rangle restricted to \mathfrak{a}_{\mathfrak{p}}$ induces an inner product on the dual space $\mathfrak{a}_{\mathfrak{p}}^{*}$ and hence also on the restricted roots.

Definition. 1) A positive restricted root $\alpha \in \Sigma$ that cannot be decomposed as a sum of two positive restricted roots is called a simple restricted root. The set of simple restricted roots is denoted by $\triangle$. 
2) An element $\lambda \in \mathfrak{a}_{\mathfrak{p}}^{*}$ is called a dominant weight (resp. strongly dominant) if $\langle\lambda, \alpha\rangle \geq 0$ (resp. $\langle\lambda, \alpha\rangle>0)$ for any $\alpha \in \triangle$.

3) Let $G$ be a complex reductive group, $T$ be a maximal torus. We call a homomorphism $\chi: T \longrightarrow$ $\mathbb{C}$ a dominant character (resp. strongly dominant) if $\operatorname{Re}\left\langle\left. d \chi\right|_{\mathfrak{a}_{\mathfrak{p}}}, \alpha\right\rangle \geq 0$ (resp. $\operatorname{Re}\left\langle\left. d \chi\right|_{\mathfrak{a}_{\mathfrak{p}}}, \alpha\right\rangle>0$ ) for any $\alpha \in \triangle$.

Example. Consider $G=\mathrm{GL}_{n}(\mathbb{C})$. Fix $T$ to be the subgroup of diagonal matrices and a Borel $B$ to be the subgroup of upper triangular matrices. Choose a compact from by the involution $A \longmapsto\left(A^{*}\right)^{-1}$ where $*$ is conjugate transpose. Under this identification we can choose $A_{\mathfrak{p}}$ to be the subgroup of real and positive diagonal matrices and $A$ to be the subgroup of real diagonal matrices. $\langle X, Y\rangle:=\operatorname{Re} \operatorname{Tr}\left(X \cdot Y^{*}\right)$ defines a real valued inner product on $\mathfrak{g l}_{n}$. Denote $E_{i j} \in \mathfrak{g l}_{n}$ to be the matrix with value 1 at the $(i, j)$ th entry and 0 at all other entries. Any character $\chi: T \longrightarrow \mathbb{C}$ can be written in the form

$$
\chi(t)=\chi\left(t_{1}, \ldots, t_{n}\right)=\prod\left|t_{i}\right|^{\lambda_{i}} \cdot\left(\frac{t_{i}}{\left|t_{i}\right|}\right)^{n_{i}},
$$

for some $\lambda_{i} \in \mathbb{C}, n_{i} \in \mathbb{Z}$. We can write $\mathfrak{g l}_{n}=\mathfrak{g}_{0} \oplus \bigoplus_{\lambda \in \Sigma} \mathfrak{g}_{\alpha_{i j}}$ where $\mathfrak{g}_{\alpha_{i j}}=\mathrm{E}_{i j}$ and $\alpha_{i j}=t_{i} / t_{j}$. Notice that the positive restricted roots are $\left\{\alpha_{i j}\right\}_{i<j}$. On the Lie algebra level, $\alpha_{i j}\left(T_{1}, \ldots, T_{n}\right)=T_{i}-$ $T_{j}$ where $T_{1}, \ldots, T_{n}$ is the corresponding basis for $\mathfrak{a}_{\mathfrak{p}}$. Note that $\left.\chi\right|_{A_{\mathfrak{p}}}(t)=\chi\left(t_{1}, \ldots, t_{n}\right)=\prod t_{i}^{\lambda_{i}}$ and hence $\left.d \chi\right|_{\mathfrak{a}_{\mathfrak{p}}}\left(T_{1}, \ldots, T_{n}\right)=\left(\lambda_{1} T_{1}, \ldots, \lambda_{n} T_{n}\right)$.

Since $\mathfrak{a}_{\mathfrak{p}}$ consists of diagonal and real matrices we have that $\operatorname{Tr}\left(X \cdot Y^{*}\right)=\sum x_{i} \cdot y_{i}$. The inner product on $\mathfrak{a}_{\mathfrak{p}}^{*}$ is defined by $\langle\alpha, \beta\rangle:=\left\langle X_{\alpha}, X_{\beta}\right\rangle$ via the identification $\alpha \longrightarrow\left\langle X_{\alpha},\right\rangle$ for $X_{\alpha} \in \mathfrak{a}_{\mathfrak{p}}$. Under this identification, $\alpha_{i j}$ corresponds to the vector $v_{i j}=(0, \ldots, 0,1,0, \ldots, 0,-1, \ldots 0)(1$ and -1 in the $i$ th and $j$ th coordinates and 0 in the others) and $\left.d \chi\right|_{\mathfrak{a}_{\mathfrak{p}}}$ corresponds to $\left(\lambda_{1}, \ldots, \lambda_{n}\right)$. Requiring $\chi$ to be dominant is the same as requiring that for any $i<j$ we have:

$$
0 \leq \operatorname{Re}\left\langle v_{i j},\left(\lambda_{1}, \ldots, \lambda_{n}\right)\right\rangle=\operatorname{Re}\left(\lambda_{i}\right)-\operatorname{Re}\left(\lambda_{j}\right) \geq 0,
$$

that is, $\operatorname{Re}\left(\lambda_{1}\right) \geq \ldots \geq \operatorname{Re}\left(\lambda_{n}\right)$. Strong dominance implies that $\operatorname{Re}\left(\lambda_{1}\right)>\ldots>\operatorname{Re}\left(\lambda_{n}\right)$.

B.1. Parabolic subgroups. We now introduce some facts about parabolic subgroups:

Definition. A parabolic subgroup of an algebraic group $\underline{G}$ defined over $k$ is a closed subgroup $\underline{P} \subseteq \underline{G}$, for which the quotient space $\underline{G} / \underline{P}$ is a projective algebraic variety. A subgroup $P$ of $G=\underline{G}(k)$ is parabolic if $P=\underline{P}(k)$ for some parabolic subgroup $\underline{P} \subseteq \underline{G}$, such that $\underline{P}(k)$ is dense in $\underline{P}$ (in the Zarisky topology).

There always exist minimal parabolic subgroups and they have the following structure theory over $\mathbb{R}$ : let $G$ be a linear connected reductive group, $K$ a maximal compact subgroup, $A_{\mathfrak{p}}, N_{\mathfrak{p}}$ as defined above and $\mathfrak{g}=\mathfrak{k} \oplus \mathfrak{p}=\mathfrak{g}_{0} \oplus \bigoplus_{\lambda \in \Sigma} \mathfrak{g}_{\lambda}$. Consider $M_{\mathfrak{p}}=C_{K}\left(\mathfrak{a}_{\mathfrak{p}}\right)$, i.e the set of $k \in K$ such that $\operatorname{Ad}_{k}=$ id on $\mathfrak{a}_{\mathfrak{p}}$. We have the following properties:

(1) $M_{\mathfrak{p}}$ is a closed subgroup of $K$, hence compact.

(2) $M_{\mathfrak{p}}$ centralizes $\mathfrak{a}_{\mathfrak{p}}$ and normalizes each $\mathfrak{g}_{\lambda}$. 
(3) $M_{\mathfrak{p}}$ centralizes $A$ and normalizes $N_{\mathfrak{p}}$.

(4) $M_{\mathfrak{p}} A_{\mathfrak{p}} N_{\mathfrak{p}}$ is a closed minimal parabolic subgroup of $G$.

(5) Every minimal parabolic subgroup of $G$ can be represented in this form (4).

Fix a minimal parabolic $Q_{\mathfrak{p}}:=M_{\mathfrak{p}} A_{\mathfrak{p}} N_{\mathfrak{p}}$. A Standard parabolic subgroup is any closed subgroup $Q$ that contains $Q_{\mathfrak{p}}$. Any standard parabolic subgroup has a Langlands decomposition of the form $Q=M A N$ obtained as follows:

- $M A:=Q \cap \Theta(Q)$, where $\Theta$ is the global Cartan involution that fixes $K$.

- $A:=Z(M A)$ with Lie algebra $\mathfrak{a}$.

- $\mathfrak{m}$ is the orthogonal complement of $\mathfrak{a}$ in $\mathfrak{m} \oplus \mathfrak{a}$ with respect to $\langle$,$\rangle defined above.$

- $M_{0}$ is the analytic subgroup that corresponds to $\mathfrak{m} . M=C_{K}(A) \cdot M_{0}$ (it is non compact if $Q \neq Q_{\mathfrak{p}}$ ).

- Let $\mathfrak{n}$ be the direct sum of eigenspaces in $\mathfrak{q}=\operatorname{Lie}(Q)$ with nonzero eigenvalues corresponds to $\mathfrak{a}$, and define $N$ to be the analytic subgroup corresponding to $\mathfrak{n}$.

This describes the structure of any standard parabolic. There is another description of standard parabolic subgroups, from which one can understand the connection between $\mathfrak{a}_{\mathfrak{p}}, \mathfrak{m}_{\mathfrak{p}}, \mathfrak{n}_{\mathfrak{p}}$ and $\mathfrak{a}, \mathfrak{m}, \mathfrak{n}$ : let $F$ be any subset of $\triangle$. Denote $\Sigma_{F}=\{\beta \in \Sigma \mid \beta \in \operatorname{Span}(F)\}, \Gamma_{F}=\Sigma^{+} \cup \Sigma_{F}$ and define:

$$
\mathfrak{q}_{F}=\mathfrak{m}_{\mathfrak{p}} \oplus \mathfrak{a}_{\mathfrak{p}} \oplus \bigoplus_{\beta \in \Gamma_{F}} \mathfrak{g}_{\beta}
$$

This is a parabolic subalgebra of $\mathfrak{g}$ containing $\mathfrak{q}_{\mathfrak{p}}=\mathfrak{m}_{\mathfrak{p}} \oplus \mathfrak{a}_{\mathfrak{p}} \oplus \mathfrak{n}_{\mathfrak{p}}$. Define:

(1) $\mathfrak{a}_{F}:=\cap_{\beta \in \Sigma_{F}} \operatorname{ker} \beta$. It is contained in $\mathfrak{a}_{\mathfrak{p}}$.

(2) $\mathfrak{a}_{M, F}:=\mathfrak{a}_{F}^{\perp} \subseteq \mathfrak{a}_{\mathfrak{p}}$.

(3) $\mathfrak{m}_{F}:=\mathfrak{a}_{M, F} \oplus \mathfrak{m}_{\mathfrak{p}} \oplus \bigoplus_{\beta \in \Sigma_{F}} \mathfrak{g}_{\beta}$.

(4) $\mathfrak{n}_{F}:=\bigoplus_{\beta \in \Sigma^{+} \backslash \Sigma_{F}} \mathfrak{g}_{\beta}$.

(5) $\mathfrak{n}_{M, F}:=\mathfrak{n}_{p} \cap \mathfrak{m}_{F}$.

and we get that $\mathfrak{q}_{F}=\mathfrak{m}_{F} \oplus \mathfrak{a}_{F} \oplus \mathfrak{n}_{F}$. This decomposition has some nice properties:

- The centralizer of $\mathfrak{a}_{F}$ in $\mathfrak{g}$ is $\mathfrak{m}_{F} \oplus \mathfrak{a}_{F}$.

- $\mathfrak{a}_{\mathfrak{p}}=\mathfrak{a}_{F} \oplus \mathfrak{a}_{M, F}$.

- $\mathfrak{n}_{\mathfrak{p}}=\mathfrak{n}_{F} \oplus \mathfrak{n}_{M, F}$.

Now let $A_{F}$ and $N_{F}$ be the analytic subgroups with Lie algebras $\mathfrak{a}_{F}, \mathfrak{n}_{F}$. Define $M_{0, F}$ to be the analytic subgroup that correspond to $\mathfrak{m}_{F}$ and $M=C_{K}\left(A_{F}\right) \cdot M_{0}$. We get a standard parabolic subgroup $Q_{F}:=M_{F} A_{F} N_{F}$ with Lie algebra $\mathfrak{q}_{F}=\mathfrak{m}_{F} \oplus \mathfrak{a}_{F} \oplus \mathfrak{n}_{F}$. This describes all standard parabolic subgroups. Notice that if $G$ is semisimple then $\operatorname{dim} A_{\mathfrak{p}}=|\triangle|$ and there are exactly $2^{\operatorname{dim} A_{\mathfrak{p}}}$ different subsets $F \subseteq \triangle$ and the number of standard parabolic subgroups is $2^{\operatorname{dim} A_{p}}$.

Example. Consider $G=\mathrm{SL}_{\mathrm{n}}(\mathbb{R})$ and fix a minimal parabolic subgroup $M_{\mathfrak{p}} A_{\mathfrak{p}} N_{\mathfrak{p}}$ corresponding to the upper triangular matrices. The standard parabolic subgroups $Q_{F}$ are block upper triangular matrices, where different choices of $F$ corresponds to different matrix block partitions. Here, 
$M_{F} A_{F}$ corresponds to block diagonal matrices. When $F$ is empty, we get that $Q_{F}=Q_{\mathfrak{p}}$, the minimal parabolic subgroup and when $F=\triangle$ we get that $Q_{F}=G, A, N=\{e\}$ and $M=G$.

\section{Appendix C. Restriction of scalars and Galois involution}

Let $k$ be a field, $\underline{G}$ be an algebraic group defined over $k$. It can be written as $\underline{G}=\operatorname{Spec}(A)$ where $A=k\left[x_{1}, \ldots, x_{n}\right] /\left\langle f_{1}, \ldots, f_{m}\right\rangle$ is a $k$-algebra. Let $K \supset k$ be a field extension and define $\underline{G}(K):=\operatorname{Hom}_{k}(A, K)$. This set is called the $K$ points of $\underline{G}$ and it is in bijection with the solutions of $\left\{f_{1}\left(x_{1}, \ldots, x_{n}\right)=0, \ldots, f_{m}\left(x_{1}, \ldots, x_{n}\right)=0\right\}$ in $K . \underline{G}(K)$ has a structure of a group induced by the group structure of $\underline{G}$. Notice that the $\mathbb{C}$ (resp. $\mathbb{R}$ )-points of an algebraic group $\underline{G}$ defined over $\mathbb{R}$ is a complex (resp. real) Lie group of the same dimension.

Now set $k=\mathbb{R}$. A real structure on $\underline{G}$ is determined by an action of $\operatorname{Gal}(\mathbb{C} / \mathbb{R})=\{\mathrm{id}, \tau\}$ on its coordinate ring $\mathbb{C}[\underline{G}]$, such that $\mathbb{C}[\underline{G}]^{\tau} \otimes_{\mathbb{R}} \mathbb{C} \simeq \mathbb{C}[\underline{G}]$. We denote $\mathbb{R}[\underline{G}]:=\mathbb{C}[\underline{G}]^{\tau}$ as the real structure. $\operatorname{Gal}(\mathbb{C} / \mathbb{R})$ also acts on $\underline{G}(\mathbb{C})$ such that $(\underline{G}(\mathbb{C}))^{\sigma}$ is isomorphic to $\operatorname{Hom}_{\mathbb{R}}(\mathbb{R}[\underline{G}], \mathbb{R})$. A motivating example is $\underline{G}=\mathrm{GL}_{n}, \mathbb{C}[\underline{G}]=\mathbb{C}\left[t_{11}, \ldots, t_{n n}, \operatorname{det}^{-1}\right], \mathbb{R}[\underline{G}]=\mathbb{R}\left[t_{11}, \ldots, t_{n n}, \operatorname{det}^{-1}\right]=\mathbb{C}[\underline{G}]^{\tau}$, where $\tau$ acts by complex conjugation.

Note that $\operatorname{Gal}(\mathbb{C} / \mathbb{R})$ acts by an involution $\tau$ on $\mathbb{C}[\underline{G}]$, but it is not a $\mathbb{C}$-algebra automorphism as it is not $\mathbb{C}$-linear. In order to view $\tau$ as a morphism of algebraic group, we need to introduce restriction of scalars.

Fact. There exists a functor $\operatorname{Res}_{\mathbb{C} / \mathbb{R}}$ (called restriction of scalars) from the category of affine group schemes over $\mathbb{C}$ to the category of affine group schemes over $\mathbb{R}$, that is right adjoint to base change, i.e:

$$
\operatorname{Hom}\left(G, \operatorname{Res}_{\mathbb{C} / \mathbb{R}}\left(G^{\prime}\right)\right) \simeq \operatorname{Hom}\left(G \times_{\operatorname{Spec} \mathbb{R}} \operatorname{Spec} \mathbb{C}, G^{\prime}\right),
$$

where $G$ and $G^{\prime}$ are algebraic groups defined over $\mathbb{R}$ and $\mathbb{C}$, respectively.

We can now apply the following procedure. Let $\underline{G}$ be an algebraic group defined over $\mathbb{R}$, with a real structure $\mathbb{C}[\underline{G}]^{\tau}$ for $\tau \in \operatorname{Gal}(\mathbb{C} / \mathbb{R})$. Consider $\underline{G}$ as an algebraic group over $\mathbb{C}$ (denoted $\underline{G}_{\mathbb{C}}$ ) and apply the functor $\operatorname{Res}_{\mathbb{C} / \mathbb{R}}$ to get an algebraic group $\underline{G}_{\mathbb{C} / \mathbb{R}}:=\operatorname{Res}_{\mathbb{C} / \mathbb{R}}\left(\underline{G}_{\mathbb{C}}\right)$ defined over $\mathbb{R}$. We have the following properties:

$* \underline{G}(\mathbb{C})=\underline{G}_{\mathbb{C} / \mathbb{R}}(\mathbb{R})$.

* The map $\tau: \underline{G} \longrightarrow \underline{G}$ induces an involution of $\mathbb{R}$-algebraic groups $\theta: \underline{G}_{\mathbb{C} / \mathbb{R}} \longrightarrow \underline{G}_{\mathbb{C} / \mathbb{R}}$ such that $\underline{G^{\theta} / \mathbb{R}}(\mathbb{R})=\underline{G}(\mathbb{R})$.

Definition. We call the involution $\theta: \underline{G}_{\mathbb{C} / \mathbb{R}} \longrightarrow \underline{G}_{\mathbb{C} / \mathbb{R}}$ defined above a Galois involution. Note that $\underline{G}_{\mathbb{C} / \mathbb{R}}(\mathbb{R}) / \underline{G}_{\mathbb{C} / \mathbb{R}}^{\theta}(\mathbb{R}) \simeq \underline{G}(\mathbb{C}) / \underline{G}(\mathbb{R})$.

Theorem C.1. Let $\underline{G}$ be an algebraic group defined over $\mathbb{R}, \underline{G}_{\mathbb{C} / \mathbb{R}}$ be the restriction of scalars of its complexification, and $\theta: \underline{G}_{\mathbb{C} / \mathbb{R}} \longrightarrow \underline{G}_{\mathbb{C} / \mathbb{R}}$ be a Galois involution. Let $G=\underline{G}_{\mathbb{C} / \mathbb{R}}(\mathbb{R})$ and $H=\underline{G}_{\mathbb{C} / \mathbb{R}}^{\theta}(\mathbb{R})$. Then $\theta: G \longrightarrow G$ has the property $(\star)$ (see Section 1.1). 
Proof. Fix a Borel subgroup $B$, a $\theta$-stable maximal torus $T$ and a $\theta$-stable maximal $\mathbb{R}$-split torus $A$, all in $G$, such that $A_{\mathfrak{p}} \subseteq A \subseteq T \subseteq B$ (see Proposition 6.1). If we consider $G$ as a complex group then we can write $\operatorname{Lie}(G)=\mathfrak{g}=\mathfrak{g}_{0} \oplus \bigoplus_{\widetilde{\alpha} \in \Sigma(G, T)} \mathfrak{g}_{\widetilde{\alpha}}$, where $\mathfrak{g}_{0}=\operatorname{Lie}(T), \Sigma(G, T)$ is the corresponding root system and each eigenspace $\mathfrak{g}_{\widetilde{\alpha}}$ is one dimensional over $\mathbb{C}$. If we consider $G$ as a real group then we can write $\mathfrak{g}=\mathfrak{g}_{0} \oplus \bigoplus_{\alpha \in \Sigma\left(G, A_{\mathfrak{p}}\right)} \mathfrak{g}_{\alpha} \cdot \mathfrak{g}_{\alpha}$ becomes a two dimensional space over $\mathbb{R}$ and $\left.\widetilde{\alpha}\right|_{A_{\mathfrak{p}}}=\alpha$. Notice that $\operatorname{dim} H=\frac{1}{2} \cdot \operatorname{dim} G$ and $\mathfrak{g}^{\theta}=\mathfrak{g}_{0}^{\theta} \oplus \bigoplus_{\alpha \in \Sigma(G, T)} \mathfrak{g}_{\alpha}$ has exactly half of the dimension in each root space.

Note that for any $n \in N_{G}(T), \mathrm{Ad}_{n}$ is a $\mathbb{C}$-linear map, and $d \theta$ is a semi-linear map. This implies that if $\theta_{n}(\alpha)=\alpha$ then $\left.d\left(\theta_{n}\right)\right|_{\mathfrak{g}_{\alpha}}=\left.\operatorname{Ad}_{n} \circ d \theta\right|_{\mathfrak{g}_{\alpha}} \neq \pm \mathrm{Id}$.

We now show that $\delta_{B^{\theta_{n}}}=\left.\delta_{B}^{1 / 2}\right|_{B^{\theta_{n}}}$ as a character of $B^{\theta_{n}}$. Notice that $\delta_{B}(b)=\left|\operatorname{det}\left(\operatorname{Ad}_{b}\right)\right|$, where $b \in B, \operatorname{Ad}_{b} \in \operatorname{GL}(\mathfrak{b})$ and $\mathfrak{b}:=\operatorname{Lie}(B)$. As modular characters are trivial on the unipotent part of $B^{\theta_{n}}$, it is enough to prove that $\left.\delta_{B^{\theta_{n}}}\right|_{T^{\theta_{n}}}=\left.\delta_{B}^{1 / 2}\right|_{T^{\theta_{n}}}$. Note that $S:=\left\{n \theta(t) n^{-1} t \mid t \in T\right\}$ is a connected component of $T^{\theta_{n}}$, so it is enough to prove that $\left.\delta_{B^{\theta_{n}}}\right|_{S}=\left.\delta_{B}^{1 / 2}\right|_{S}$. As $T$ is $\mathbb{C}$-split, and by the fact that modular characters are trivial on compact subgroups, it is enough to prove that $\left.\delta_{B^{\theta_{n}}}\right|_{\widetilde{S}}=\left.\delta_{B}^{1 / 2}\right|_{\widetilde{S}}$ where $\widetilde{S}=\left\{n \theta(a) n^{-1} a \mid a \in A_{\mathfrak{p}}\right\}$. Denote

$$
\begin{aligned}
& S_{1}:=\left\{\alpha \in \Sigma^{+}\left(G, A_{\mathfrak{p}}\right) \text { s.t } \alpha \neq \theta_{n}(\alpha) \in \Sigma^{+}\left(G, A_{\mathfrak{p}}\right)\right\}, \\
& S_{2}:=\left\{\alpha \in \Sigma^{+}\left(G, A_{\mathfrak{p}}\right) \text { s.t } \alpha=\theta_{n}(\alpha) \in \Sigma^{+}\left(G, A_{\mathfrak{p}}\right)\right\},
\end{aligned}
$$

and

$$
S_{3}:=\left\{\alpha \in \Sigma^{+}\left(G, A_{\mathfrak{p}}\right) \text { s.t } \theta_{n}(\alpha) \in \Sigma^{-}\left(G, A_{\mathfrak{p}}\right)\right\}
$$

Note that for any $a \in A_{\mathfrak{p}}$,

$$
\delta_{B}(a)=\left|\operatorname{det}\left(\operatorname{Ad}_{a}\right)\right|=\left|\prod_{\alpha \in \Sigma^{+}\left(G, A_{\mathfrak{p}}\right)}(\alpha(a))^{2}\right|,
$$

and therefore

$$
\delta_{B}^{1 / 2}\left(a \theta_{n}(a)\right)=\left|\prod_{\alpha \in \Sigma^{+}\left(G, A_{\mathfrak{p}}\right)} \alpha(a) \cdot \theta_{n}(\alpha)(a)\right| .
$$

$\operatorname{Lie}\left(B^{\theta_{n}}\right)$ consists of the +1 eigenspace of $\mathfrak{b}$ under $d\left(\theta_{n}\right)$, hence it is clear that roots in $S_{3}$ doesn't contribute to $\operatorname{Lie}\left(B^{\theta_{n}}\right)$, that is, $\operatorname{Lie}\left(B^{\theta_{n}}\right)=\left(\mathfrak{g}_{0} \oplus \bigoplus_{\alpha \in S_{1}, S_{2}} \mathfrak{g}_{\alpha}\right)_{d\left(\theta_{n}\right),+1}$, where ()$_{d\left(\theta_{n}\right),+1}$ denote the +1 eigenspace of $d\left(\theta_{n}\right)$. As $\left.d\left(\theta_{n}\right)\right|_{\mathfrak{g}_{\alpha}} \neq \pm \mathrm{Id}$ for roots $\alpha \in S_{2}$ we deduce that:

$$
\delta_{B^{\theta_{n}}}\left(a \theta_{n}(a)\right)=\left|\prod_{\alpha \in S_{1}, S_{2}}\left(\alpha(a) \cdot \theta_{n}(\alpha)(a)\right)\right| .
$$

Hence, it is enough to prove that $\prod_{\alpha \in S_{3}} \alpha(a) \cdot \theta_{n}(\alpha)(a)$ is trivial for any $a \in A_{\mathfrak{p}}$, or equivalently by taking differentials, $\sum_{\alpha \in S_{3}} \alpha(X)+\theta_{n}(\alpha)(X)=0$ for any $X \in \mathfrak{a}_{\mathfrak{p}}=\operatorname{Lie}\left(A_{\mathfrak{p}}\right)$. Indeed, if $\alpha \in S_{3}$ then also $-\theta_{n}(\alpha) \in S_{3}$ as well, hence we are done. 


\section{REFERENCES}

[AG08] A. Aizenbud, D. Gourevitch, Schwartz functions on Nash Manifolds, International Mathematics Research Notes (2008).

[AG09] A. Aizenbud, D. Gourevitch, Generalized Harish-Chandra descent Gelfand pairs and an Archimedean analog of Jacquet-Rallis' Theorem. Duke Mathematical Journal, Volume 149, Number 3 (2009). See also arxiv:0812.5063v3[math.RT].

[AGM16] A. Aizenbud, D. Gourevitch, A. Minchenko, Holonomicity of spherical characters and applications to multiplicity bounds, Selecta Mathematica (2016). See also arxiv 1501.01479.

[ALOF12] E.Lapid, O.Offen, B.Feigon, On representations distinguished by unitary groups, and in particular appendix B (by A. Aizenbud and E. Lapid), Publ. Math. Inst. Hautes (2012).

[Ban87] E. van den Ban, Asymptotic behaviour of matrix coefficients related to reductive symmetric spaces, Nederl. Akad. Wetensch. Indag. Math. 49 (1987), no. 3, 225-249.

[BK14] J. Bernstein and B. Krötz: Smooth Fréchet globalizations of Harish-Chandra modules. Israel J. Math. 199(1), 45-111 (2014).

[Del10] Patrick Delorme. Constant term of smooth H $\psi$-spherical functions on a reductive p-adic group. In: Transactions of the American Mathematical Society 362 (2010), pp. 933-955.

[GK75] I. M. Gelfand and D. A. Kajdan, Representations of the group GL $(n, \mathrm{~K})$ where $\mathrm{K}$ is a local field, Lie groups and their representations (Proc. Summer School, Bolyai J'anos Math. Soc., Budapest, 1971), Halsted, New York, 1975, pp. 95-118. MR MR0404534 (53 \#8334).

[GSS15] D. Gourevitch, S. Sahi, and E. Sayag, Invariant functionals on the Speh representations, Transformation groups 20 (2015), no. 4, 1023-1042. MR 3416438.

[Hum72] Humphreys, J: Introduction to Lie Algebras and Representation Theory. Graduate Texts in Mathematics, (1972).

[HW93] A. G. Helminck and S. P. Wang: On rationality properties of involutions of reductive groups, Adv. Math. 99 (1993), 26-97.

[Kem15] A. Kemarsky, Gamma Factors of Distinguished Representations of GL(n, C), Pacific J. Math. 278 (2015), no. 1, 137-172. MR 3404670.

[Kn96] A.W. Knapp, Lie groups: Beyond an introduction. Birkh“auser, Boston, MA, (1996).

[Kn01] A.W. Knapp, Representation theory of semisimple groups. An overview based on examples, Reprint of the 1986 original. Princeton Landmarks in Mathematics. Princeton University Press, Princeton, NJ, (2001).

[KO13] T. Kobayashi and T. Oshima, Finite multiplicity theorems for induction and restriction, Advances in Math. 248 (2013), 921-944.

[KS16] Bernhard Krotz and Henrik Schlichtkrull. Multiplicity bounds and the subrepresentation theorem for real spherical spaces. In: Transactions of the American Mathematical Society 368 (2016), pp. 2749-2762. arXiv: 1309.0930 [math.RT].

[KT00] Knapp, A. ,Trapa, P: Representations of semisimple Lie groups. IAS/Park City Mathematics Series 8, Amer. Math. Soc., 2000.

[Lan73] Langlands, R. P., On classification of irreducible representations of real algebraic groups. Unpublished Manuscript, 1973.

[Pra] D. Prasad, A "relative" local Langlands correspondence, preprint, 2012, available at http://www.math.tifr.res.in/ dprasad/relative-L.pdf.

[Sp85] T. A. Springer, Some results on algebraic groups with involutions, in: Algebraic groups and related topics, 525-543, Advanced Studies in Pure Math. 6, Kinokuniya-North Holland (1985).

[SV] Yiannis Sakellaridis and Akshay Venkatesh. Periods and harmonic analysis on spherical varieties. arXiv: 1203.0039 [math.RT]. 
[ZN66] D.P. Zhelobenko, M. A. Naimark Description of completely irreducible representations of a semi-simple complex Lie group. 5 (Russian) Dokl. AN SSSR 171, 1966, 25-28. English translation: Soviet Math. Dokl. 7 (1966), 1403-1406.

Faculty of Mathematics and Computer Science, Weizmann Institute of Science, 234 Herzl Street, Rehovot 76100, IsRael.

E-mail address: itay.glazer@weizmann.ac.il 